\title{
A new weighting method for improving the WENO-Z scheme
}

\author{
Shengping Liu ${ }^{1,2}$ (i) | Yiqing Shen ${ }^{1,2} \mid$ Fangjun Zeng ${ }^{1,2} \mid$ Ming $\mathrm{Yu}^{3}$
}

\author{
${ }^{1}$ State Key Laboratory of High \\ Temperature Gas Dynamics, Institute of \\ Mechanics, Chinese Academy of Sciences, \\ Beijing, China \\ ${ }^{2}$ School of Engineering Science, \\ University of Chinese Academy of \\ Sciences, Beijing, China \\ ${ }^{3}$ Institute of Applied Physics and \\ Computational Mathematics, Beijing, \\ China
}

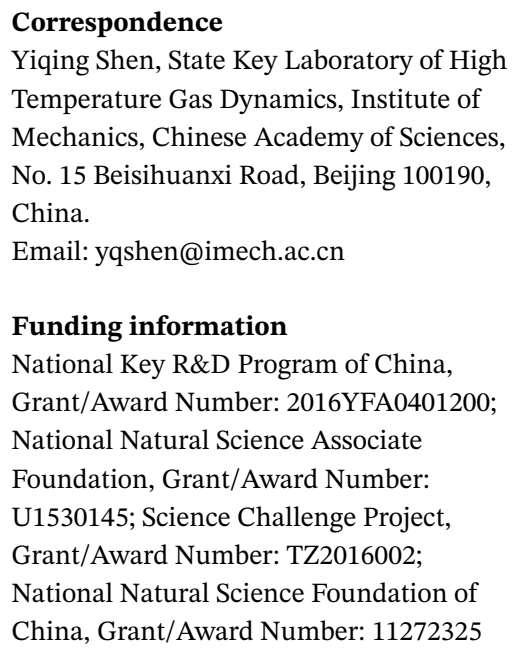

\begin{abstract}
Summary
The calculation of the weight of each substencil is very important for a weighted essentially nonoscillatory (WENO) scheme to obtain high-order accuracy in smooth regions and keep the essentially nonoscillatory property near discontinuities. The weighting function introduced in the WENO-Z scheme provides a straightforward method to analyze the accuracy order in smooth regions. In this paper, we construct a new sixth-order global smoothness indicator (GSI-6) and a function about GSI-6 and the local smoothness indicators $\left(I S_{k}\right)$ to calculate the weights. The analysis and numerical results show that, with the new weights, the scheme satisfies the sufficient condition for the fifth-order convergence in smooth regions even at critical points. Meanwhile, it can also maintain low dissipation for discontinuous solutions due to relative large weights assigned to discontinuous substencils.
\end{abstract}

\section{KEYWORDS}

global smoothness indicator, low numerical dissipation, weighting function, WENO-Z scheme

\section{1 | INTRODUCTION}

The weighted essentially nonoscillatory (WENO) schemes have now become a kind of popular method in computational fluid dynamics. ${ }^{1}$ The basic idea of WENO schemes is that the numerical flux is calculated by using a convex combination of all the local numerical fluxes of candidate substencils, each being assigned a nonlinear weight that determines the contribution of this substencil. The weights can be designed as follows. In smooth regions, they approach certain optimal weights to achieve a high order of accuracy, whereas in regions near discontinuities, the substencils that contain the discontinuities are assigned small weights to keep the property of essential nonoscillation. Hence, an important issue for developing WENO schemes is how to evaluate the smoothness of substencils and weights.

In the first WENO scheme, Liu et $\mathrm{al}^{2}$ used a linear combination of the squares of all hierarchical undivided differences to measure the smoothness of a numerical flux on a substencil. Later, the analysis of Jiang and Shu ${ }^{3}$ showed that the WENO scheme constructed from an $r$ th-order essentially nonoscillatory (ENO) scheme ${ }^{4}$ with the smoothness measurement of Liu et al is only $(r+1)$ th-order accurate, and a classical way, which uses a sum of the $L^{2}$ norms of all the derivatives of the interpolation polynomial over the interval of integration, was proposed to calculate the local smoothness indicators 


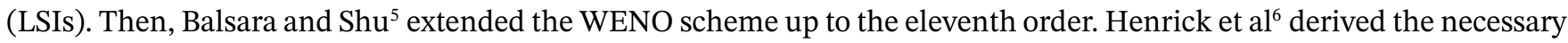
and sufficient conditions for the fifth-order convergence of a fifth-order WENO scheme and pointed out that the weights generated by the method of Jiang and $\mathrm{Shu}^{3}$ cannot meet the conditions at a critical point where the first derivative is zero. In their work, ${ }^{6}$ a mapping function for the weights was proposed and the new mapped WENO (WENO-M) scheme can achieve fifth-order convergence in smooth regions including critical points.

Borges et $\mathrm{al}^{7}$ proposed a different method to calculate weights by incorporating the higher-order information (global smoothness indicator, abbreviated to GSI) about the regularity of the numerical solution. The new weights can satisfy the sufficient conditions for optimal order. In fact, the convergence order of the fifth-order WENO (WENO-Z) scheme proposed by Borges et $\mathrm{al}^{7}$ at critical points is influenced by the power parameter in the definition of weights, for example, the accuracy is of fourth and fifth order if the power $q$ takes the value of 1 and 2, respectively. On the other hand, Borges et al pointed out that, for solutions containing the discontinuities, increasing $q$ makes the scheme more dissipative. The weighting method proposed by Borges et al provides a straightforward way for improving the performance of a WENO scheme. Castro et $\mathrm{al}^{8}$ developed higher-order WENO-Z schemes. Ha et $\mathrm{al}^{9}$ introduced a set of different LSIs for substencils and a sixth-order GSI $\zeta$ to calculate weights; the new WENO-Z-type (WENO-NS) scheme improves the ability of detecting the complicated solution structures. Kim et $\mathrm{a}^{10}$ further introduced a parameter to make a balanced trade-off among the new LSIs of $\mathrm{Ha}$ et $\mathrm{al}^{9}$; the resulted scheme (WENO-P) yields better results than WENO-NS for problems including discontinuities. Fan et $\mathrm{al}^{11}$ constructed several higher-order (up to the eighth order) GSIs; the associated WENO-Z-type schemes (WENO-Z $\eta$ ) can obtain fifth convergence order in smooth regions, even at the second-order critical points where both the first and second derivatives vanish. $\mathrm{Hu}$ et al ${ }^{12}$ constructed an adaptive central-upwind WENO scheme (WENO-CU6) in which a tunable parameter was introduced for the weighting function of WENO-Z to increase the contribution of optimal weights. Acker et $\mathrm{al}^{13}$ presented a way of increasing the relevance of less smooth substencils by adding a new term into the WENO-Z weights; the new scheme (WENO-Z+) improves the resolution of the high-frequency smooth waves. These WENO-Z-type schemes mentioned above successfully improve the accuracy and decrease the dissipation in many problems. However, some of them, such as WENO-NS, WENO-P, WENO-CU6, and WENOZ+ schemes, introduced empirical tunable parameters, and hence, their applications are limited. Some of them, such as WENO-Z $\eta$ schemes, are prone to generate spurious oscillations near shock regions. Although the authors ${ }^{7,13}$ demonstrated that the smaller dissipation of the scheme has more influence than its convergence rate at critical points when solving problems with shocks; how to get both the optimal order at critical points and lower the dissipation of a WENO-Z-type scheme is still worthy to be studied, especially for simulating the complicated flow fields.

In this paper, we propose a new sixth-order GSI (GSI-6), and a function consisted of the GSI-6 and LSIs ( $I S_{k}$ ) to calculate the weights of the fifth-order WENO scheme. For a smooth solution, the new weights satisfy the sufficient condition for fifth-order convergence in smooth regions; hence, the new scheme performs better than the WENO-Z scheme. However, for discontinuous solutions, the weights of the new scheme assigned to discontinuous substencils are as large as the ones of WENO-Z, ie, the behavior of the new scheme in shock regions is similar to that of the WENO-Z scheme. Hence, the new scheme performs well for both smooth and discontinuous solutions.

This paper is organized as follows. In Section 2, several WENO schemes are briefly introduced. In Section 3, the new scheme is proposed and a detailed discussion is given. In Section 4, various numerical examples are presented to demonstrate the good performance of the new scheme. Concluding remarks are given in Section 5.

\section{2 | THE WENO SCHEMES}

In this section, we briefly review the WENO schemes by using the scalar conservative law equation

$$
\frac{\partial u}{\partial t}+\frac{\partial f(u)}{\partial x}=0
$$

As in the works of Henrick et $\mathrm{al}^{6}$ and Borges et al, ${ }^{7}$ by defining the points $x_{i}=i \Delta x,(i=0, \ldots, N)$, where $\Delta x$ is the uniform grid spacing, (1) can be approximated by a conservative finite difference formula as follows:

$$
\frac{d u_{i}}{d t}=-\frac{\hat{f}_{i+1 / 2}-\hat{f}_{i-1 / 2}}{\Delta x}
$$

where $\hat{f}_{i \pm 1 / 2}$ are the numerical fluxes. 


\section{1 | The WENO-JS scheme}

The flux of a fifth-order $\mathrm{WENO}^{3}$ scheme can be written as

$$
\hat{f}_{i+1 / 2}=\sum_{k=0}^{2} \omega_{k} q_{k}
$$

where $q_{k}$ is the third-order flux on the substencil $S_{k}=(i+k-2, i+k-1, i+k)$ and given by

$$
\left\{\begin{array}{l}
q_{0}=\frac{1}{3} f_{i-2}-\frac{7}{6} f_{i-1}+\frac{11}{6} f_{i} \\
q_{1}=-\frac{1}{6} f_{i-1}+\frac{5}{6} f_{i}+\frac{1}{3} f_{i+1} \\
q_{2}=\frac{1}{3} f_{i}+\frac{5}{6} f_{i+1}-\frac{1}{6} f_{i+2}
\end{array}\right.
$$

The weight function of Jiang and $\mathrm{Shu}^{3}$ is

$$
\omega_{k}^{J S}=\frac{\alpha_{k}}{\alpha_{0}+\alpha_{1}+\alpha_{2}}, \alpha_{k}=\frac{c_{k}}{\left(I S_{k}+\epsilon\right)^{2}}, k=0,1,2,
$$

where $I S_{k}$ is called as LSI, which is used to measure the relative smoothness of a solution on the substencil $S_{k}$. $c_{0}=$ $0.1, c_{1}=0.6$ and $c_{2}=0.3$ are the optimal weights, which generate the fifth-order upstream scheme. $\epsilon$ is a positive real number introduced to avoid the denominator becoming zero. Henrick et $\mathrm{al}^{6} \mathrm{discussed}$ the role of $\epsilon$. To avoid the values of $\epsilon$ affecting the ENO behavior, $\epsilon=10^{-40}$ is suggested by Henrick et $\mathrm{al}^{6}$ and used in this paper.

Jiang and $\mathrm{Shu}^{3}$ proposed a classical LSI as

$$
I S_{k}=\sum_{l=1}^{r-1} \int_{x_{i-1 / 2}}^{x_{i+1 / 2}}(\Delta x)^{2 l-1}\left(q_{k}^{(l)}\right)^{2} d x
$$

where $q_{k}^{(l)}$ is the $l$ th-order derivative of $q_{k}(x)$ and $q_{k}(x)$ is the interpolation polynomial on substencil $S_{k}$. Shen and Zha ${ }^{14}$ suggested using a flexible and simple formula to calculate the LSI

$$
I S_{k}=\sum_{l=1}^{r-1} \gamma_{l}\left[f_{k}^{(l)}\right]^{2}
$$

where $f_{k}^{(l)}$ is the approximation of the $l$ th derivative $f_{x_{i}}^{(l)} \Delta x^{l}$ on substencil $S_{k}$. For the fifth-order WENO scheme, $r=3$ and

$$
\left\{\begin{array} { l } 
{ f _ { 0 } ^ { ( 1 ) } = ( f _ { i - 2 } - 4 f _ { i - 1 } + 3 f _ { i } ) / 2 , } \\
{ f _ { 1 } ^ { ( 1 ) } = ( - f _ { i - 1 } + f _ { i + 1 } ) / 2 , } \\
{ f _ { 2 } ^ { ( 1 ) } = ( - 3 f _ { i } + 4 f _ { i + 1 } - f _ { i + 2 } ) / 2 , }
\end{array} \text { and } \quad \left\{\begin{array}{l}
f_{0}^{(2)}=f_{i-2}-2 f_{i-1}+f_{i}, \\
f_{1}^{(2)}=f_{i-1}-2 f_{i}+f_{i+1}, \\
f_{2}^{(2)}=f_{i}-2 f_{i+1}+f_{i+2} .
\end{array}\right.\right.
$$

The coefficients $\gamma_{1}$ and $\gamma_{2}$ can choose different values. For example, the formula (6) of Jiang and Shu gives $\gamma_{1}=1$ and $\gamma_{2}=13 / 12$; Fan et al ${ }^{11}$ used $\gamma_{1}=1$ and $\gamma_{2}=1$. For analyzing conveniently in the next content, the Taylor expansion of $I S_{k}$ at $x_{i}$ for a smooth solution is given as the following:

$$
\left\{\begin{array}{l}
I S_{0}=f_{i}^{\prime 2} \Delta x^{2}+\left(\frac{13}{12} f_{i}^{\prime \prime 2}-\frac{2}{3} f_{i}^{\prime} f_{i}^{\prime \prime \prime}\right) \Delta x^{4}+\left(-\frac{13}{6} f_{i}^{\prime \prime} f_{i}^{\prime \prime \prime}+\frac{1}{2} f_{i}^{\prime} f_{i}^{(4)}\right) \Delta x^{5}+O\left(\Delta x^{6}\right) \\
I S_{1}=f_{i}^{\prime 2} \Delta x^{2}+\left(\frac{13}{12} f_{i}^{\prime \prime 2}+\frac{1}{3} f_{i}^{\prime} f_{i}^{\prime \prime \prime}\right) \Delta x^{4}+O\left(\Delta x^{6}\right) \\
I S_{2}=f_{i}^{\prime 2} \Delta x^{2}+\left(\frac{13}{12} f_{i}^{\prime \prime 2}-\frac{2}{3} f_{i}^{\prime} f_{i}^{\prime \prime \prime}\right) \Delta x^{4}+\left(\frac{13}{6} f_{i}^{\prime \prime} f_{i}^{\prime \prime \prime}-\frac{1}{2} f_{i}^{\prime} f_{i}^{(4)}\right) \Delta x^{5}+O\left(\Delta x^{6}\right)
\end{array}\right.
$$

The expansion (9) means that

$$
I S_{k}= \begin{cases}D_{0}\left[1+O\left(\Delta x^{2}\right)\right], D_{0}=f_{i}^{\prime 2} \Delta x^{2}, & f^{\prime} \neq 0 \\ D_{1}[1+O(\Delta x)], D_{1}=\frac{13}{12} f_{i}^{\prime \prime} \Delta x^{4}, & f^{\prime}=0\end{cases}
$$

Substituting (10) into (5) gives

$$
\omega_{k}^{J S}= \begin{cases}c_{k}+O\left(\Delta x^{2}\right), & f^{\prime} \neq 0, \\ c_{k}+O(\Delta x), & f^{\prime}=0 .\end{cases}
$$


Henrick et $\mathrm{l}^{6}$ derived the necessary and sufficient conditions for the fifth-order convergence of a fifth-order WENO scheme and pointed out that the WENO-JS scheme may even decrease to third-order accuracy at critical points; hence, a mapping function ${ }^{6}$ is proposed to make the new weights to satisfy a sufficient condition, which is given as

$$
\omega_{k}=c_{k}+O\left(\Delta x^{3}\right)
$$

Although it is not necessary, as mentioned by Henrick et al, ${ }^{6}(12)$ can serve as a simple criteria to design the weights for fifth-order WENO schemes.

\section{2 | The WENO-Z scheme}

The fifth-order WENO-Z scheme is proposed by Borges et $\mathrm{al}^{7}$ by introducing a GSI $\tau$ to calculate the weights

$$
\omega_{k}^{Z}=\frac{\alpha_{k}}{\alpha_{0}+\alpha_{1}+\alpha_{2}}, \quad \alpha_{k}=c_{k}\left(1+\left(\frac{\tau}{I S_{k}+\epsilon}\right)^{q}\right) .
$$

The original GSI of Borges et al is

$$
\tau_{5}=\left|I S_{2}-I S_{0}\right|
$$

Applying the Taylor expansion of $I S_{k}(9)$, there is

$$
\tau_{5}=\left|\frac{13}{3} f^{\prime \prime} f^{\prime \prime \prime}-f^{\prime} f^{(4)}\right| \Delta x^{5}+O\left(\Delta x^{6}\right) .
$$

Hence, one can get

$$
\omega_{k}^{Z}= \begin{cases}c_{k}+O\left(\Delta x^{3 q}\right), & f_{i}^{\prime} \neq 0, \\ c_{k}+O\left(\Delta x^{q}\right), & f_{i}^{\prime}=0,\end{cases}
$$

where $q$ is a tunable parameter. The numerical results in the work of Borges et $\mathrm{al}^{7}$ demonstrated that, if $q$ takes 1 , the accuracy order at critical points is only fourth; with $q=2$, the scheme can achieve fifth-order accuracy. Meanwhile, Borges et al pointed out that, for a solution containing the discontinuities, increasing $q$ makes the scheme more dissipative and the smaller dissipation of WENO-Z has much more influence than its rate of convergence at critical points when solving problems with shocks. Hence, $q=1$ is suggested for the WENO-Z scheme in the work of Borges et al. ${ }^{7}$

\section{3 | Several improved WENO-Z-type schemes}

The weight function (13) of the WENO-Z scheme provides a straightforward method for improving the accuracy of a WENO scheme. Here, several improved WENO-Z-type schemes are briefly introduced.

WENO-NS: Ha et $\mathrm{al}^{9}$ constructed a sixth-order GSI as

$$
\zeta=\frac{1}{2}\left(\left|\beta_{0}-\beta_{2}\right|^{2}+g\left(\left|L_{1,1} f\right|\right)^{2}\right), g(x)=\frac{x^{3}}{1+x^{3}},
$$

where $\beta_{k}=\xi\left|L_{1, k} f\right|+\left|L_{2, k} f\right|$ takes the similar expression as (7) in which $\xi$ is a tunable parameter and $L_{l, k} f$ is the approximation of the $l$ th derivative $f_{i+1 / 2}^{(l)}$ on substencil $S_{k}$ and the LSI $I S_{k}$ is calculated as $I S_{k}=\beta_{k}^{2}$.

WENO-P: To save the computational cost, Kim et $\mathrm{l}^{10}$ simplified the sixth-order GSI (17) as

$$
\zeta=\left(\beta_{0}-\beta_{2}\right)^{2}
$$

and introduced a parameter $\delta$ to make a balanced contribution of the $\beta_{k}$ of $\mathrm{Ha}$ et al as

$$
\tilde{\beta}_{0}=\beta_{0}, \quad \tilde{\beta}_{1}=(1+\delta) \beta_{1}, \tilde{\beta}_{2}=(1-\delta) \beta_{2} .
$$

WENO-Z $\eta$ : Fan et $\mathrm{al}^{11}$ proposed a sixth-order GSI as

$$
\tau_{6}=\left|\eta_{5}-\frac{I S_{0}+4 I S_{1}+I S_{2}}{6}\right|
$$

and 2 eighth-order GSIs as

$$
\begin{aligned}
& \tau_{81}=\left|\left(\left|f_{0}^{(1)}\right|-\left|f_{2}^{(1)}\right|\right)\left(f_{0}^{(2)}+f_{2}^{(2)}-2 f_{1}^{(2)}\right)\right|, \\
& \tau_{82}=\left(\left|f_{0}^{(1)}\right|-\left|f_{2}^{(1)}\right|\right)^{2}+\left(f_{0}^{(2)}+f_{2}^{(2)}-2 f_{1}^{(2)}\right)^{2},
\end{aligned}
$$

where $\eta_{5}=\frac{1}{144}\left[\left(f_{i-2}-8 f_{i-1}+8 f_{i+1}-f_{i+2}\right)^{2}+\left(f_{i-2}-16 f_{i-1}+30 f_{i}-16 f_{i+1}+f_{i+2}\right)^{2}\right]$ and the $I S_{k}(7)$ with $\gamma_{1}=\gamma_{2}=1$ is used. 
WENO-Z+: Acker et $\mathrm{al}^{13}$ proposed a way of improving the results of WENO-Z by increasing the weights of less smooth substencils

$$
\alpha_{k}=c_{k}\left[1+\left(\frac{\tau_{5}+\epsilon}{I S_{k}+\epsilon}\right)^{2}+\lambda\left(\frac{I S_{k}+\epsilon}{\tau_{5}+\epsilon}\right)\right],
$$

where $\lambda$ is a parameter being dependent on the grid spacing.

WENO-CU6: $\mathrm{Hu}$ et $\mathrm{al}^{12}$ constructed an adaptive central-upwind WENO scheme in which the weights are constructed as

$$
\omega_{k}=\frac{\alpha_{k}}{\sum_{k=0}^{k=3} \alpha_{k}}, \alpha_{k}=c_{k}\left(C+\frac{\tau_{6}}{I S_{k}+\epsilon}\right),
$$

where $I S_{3}$ is the smoothness indicator (6) on the stencil $S^{6}=(i-2, \ldots, i+3)$ and $\tau_{6}=\left|I S_{3}-\left(I S_{0}+4 I S_{1}+I S_{2}\right) / 6\right|$. The parameter $C\left(C=20\right.$ was suggested in the work of $\left.\mathrm{Hu}_{\mathrm{et}} \mathrm{al}^{12}\right)$ is introduced to increase the contribution of optimal weights and decrease the numerical dissipation.

Numerical results showed that the aforementioned improved WENO-Z-type schemes perform well in most of the tested cases. However, those parameters, such as $\xi$ in (17) and (18), $\delta$ in (19), $C$ in (23), and $\lambda$ in (22), are problem dependent and chosen empirically, and the WENO-Z $\eta$ schemes (use high-order GSIs (21) are prone to generate oscillations near discontinuities. Hence, the applications of these schemes to complex flow fields are greatly limited.

\section{3 | THE NEW WENO SCHEME}

In this section, we propose a new method to improve the accuracy of the WENO-Z scheme at critical points while keeping the ENO property near discontinuities.

\section{1 | The new weighting method}

First, a new GSI is designed as

$$
\tau_{6}^{\prime}=\gamma_{1}\left(\left|f_{0}^{(1)}\right|-\left|f_{2}^{(1)}\right|\right)^{2}+\gamma_{2}\left(\left|f_{0}^{(2)}\right|-\left|f_{2}^{(2)}\right|\right)^{2},
$$

where the parameters $\gamma_{1}$ and $\gamma_{2}$ are the same as used for $I S_{k}(7)$; in this paper, they take the same values $\left(\gamma_{1}=1\right.$ and $\gamma_{2}=13 / 12$ ) as those of Jiang and Shu. ${ }^{3}$ It should be pointed out, since $f_{k}^{(l)}$ is required to calculate LSI $I S_{k}(7), \tau_{6}^{\prime}$ has only a few operations more than $\tau_{5}(14)$.

Then, a new method for calculating the weights is proposed as

$$
\omega_{k}^{Z A}=\frac{\alpha_{k}}{\alpha_{0}+\alpha_{1}+\alpha_{2}}, \alpha_{k}=c_{k}\left(1+\frac{A \cdot \tau_{6}^{\prime}}{I S_{k}+\epsilon}\right),
$$

where the function $A$ is suggested as

$$
A=\frac{\tau_{6}^{\prime}}{I S_{0}+I S_{2}-\tau_{6}^{\prime}+\epsilon}
$$

Clearly, there is

$$
\left.\left.\tau_{6}^{\prime} \leq \gamma_{1}\left[f_{0}^{(1)}\right]^{2}+\gamma_{2}\left[f_{0}^{(2)}\right)\right]^{2}+\gamma_{1}\left[f_{2}^{(1)}\right]^{2}+\gamma_{2}\left[f_{2}^{(2)}\right)\right]^{2}=I S_{0}+I S_{2} .
$$

Hence, $I S_{0}+I S_{2}-\tau_{6}^{\prime} \geq 0$ and $A>0$.

\section{2 | The properties of the new weights}

Next, we discuss the accuracy of the new weights in smooth regions and their behaviors near discontinuities.

For a smooth global stencil, by means of the Taylor series expansion and simple derivation, one can get

$$
\begin{aligned}
\tau_{6}^{\prime} \leq \gamma_{1}\left(f_{0}^{(1)}-f_{2}^{(1)}\right)^{2}+\gamma_{2}\left(f_{0}^{(2)}-f_{2}^{(2)}\right)^{2} & =\left(\frac{1}{4} f_{i}^{(4)}(\Delta x)^{4}+O(\Delta x)^{6}\right)^{2}+\left(\frac{13}{6} f_{i}^{\prime \prime \prime}(\Delta x)^{3}+O(\Delta x)^{5}\right)^{2} \\
& =O\left(\Delta x^{6}\right) .
\end{aligned}
$$


Then, substituting (9) and (28) into (26), one can easily find that

$$
A= \begin{cases}O\left(\Delta x^{4}\right), & f_{i}^{\prime} \neq 0, \\ O\left(\Delta x^{2}\right), & f_{i}^{\prime}=0 .\end{cases}
$$

Using the definition of the weights (25), it is obtained that

$$
\omega_{k}^{Z A}=c_{k}+O\left(\frac{A \cdot \tau_{6}^{\prime}}{I S_{k}+\epsilon}\right)= \begin{cases}c_{k}+O\left(\Delta x^{8}\right) & , f_{i}^{\prime} \neq 0, \\ c_{k}+O\left(\Delta x^{4}\right) & , f_{i}^{\prime}=0 .\end{cases}
$$

Formula (30) implies that the new weights go beyond the sufficient condition (12), and hence, the fifth-order accuracy is guaranteed, including at critical points $\left(f_{i}^{\prime}=0\right)$.

If the solution is discontinuous on a global stencil, without loss of generality, assume that the substencil $S_{0}$ contains the discontinuity and $S_{2}$ is smooth; then, there is $I S_{0} \gg I S_{2}$, and

$$
\tau_{6}^{\prime} \approx I S_{0} \gg I S_{2} \text { and } A \gg 1 \text {. }
$$

Thus, $\alpha_{k}$ in (25) can be approximated by

$$
\alpha_{k}=A \cdot c_{k}\left(\frac{1}{A}+\frac{\tau_{6}^{\prime}}{I S_{k}+\epsilon}\right) \simeq A \cdot \tau_{6}^{\prime}\left(\frac{c_{k}}{I S_{k}+\epsilon}\right) .
$$

That means the regularized weights $\left(\omega_{k}^{Z A}\right)$ are mainly determined by the LSI $I S_{k}$.Hence, the ENO property of the new scheme can be preserved well.

In addition, using the expression of function $A(26), \alpha_{k}$ in (25) can also be written as

$$
\alpha_{k}=c_{k}\left(1+\left(\frac{I S_{k}+\epsilon}{I S_{0}+I S_{2}-\tau_{6}^{\prime}+\epsilon}\right)\left(\frac{\tau_{6}^{\prime}}{I S_{k}+\epsilon}\right)^{2}\right) .
$$

For a smooth stencil,

$$
\frac{I S_{k}+\epsilon}{I S_{0}+I S_{2}-\tau_{6}^{\prime}+\epsilon}=\left\{\begin{array}{l}
\frac{1}{2}+O\left(\Delta x^{4}\right), f_{i}^{\prime} \neq 0, \\
\frac{1}{2}+O\left(\Delta x^{2}\right), f_{i}^{\prime}=0 .
\end{array}\right.
$$

Hence, the new scheme has the same accuracy order (fifth order) as the weighted scheme with

$$
\alpha_{k}=c_{k}\left(1+\left(\frac{\tau_{6}^{\prime}}{I S_{k}+\epsilon}\right)^{2}\right) .
$$

From the aforementioned analysis, we can conclude that the new scheme has low dissipation as the WENO-Z-type scheme with $q=1$ on the discontinuous stencil and is fifth-order convergence as the WENO-Z-type scheme with $q=2$ on the smooth stencil. For convenience, the WENO-Z-type scheme with $\tau_{6}^{\prime}(24)$ is denoted as WENO-Z $\tau_{6}^{\prime}$ and the new scheme (25) is denoted as WENO-ZA.

It is noteworthy that the self-adaptive function $A$ is very different from the tuning parameter $C \cdot{ }^{12}$ For smooth solutions, $A$ is much smaller than $1(A \ll 1)$ and it plays a similar role in the weights as a large constant $C$ introduced in (23) does; however, for solutions containing discontinuities, large $C$ may generate oscillation and instability ${ }^{12}$ whereas $A$ is much larger than $1(A \gg 1)$ and its effect similar to a small $C$, as shown in (32); hence, the ENO properties can be preserved well.

As a matter of fact, $\left(A \cdot \tau_{6}^{\prime}\right)$ can be regarded as a GSI $\tau_{8}^{\prime}$, which is independent of $k$ and has the same dimension as $I S_{k}$. From (28) and (29), there is

$$
\tau_{8}^{\prime}=A \cdot \tau_{6}^{\prime}= \begin{cases}O\left(\Delta x^{10}\right), & f_{i}^{\prime} \neq 0, \\ O\left(\Delta x^{8}\right), & f_{i}^{\prime}=0 .\end{cases}
$$

\subsection{The dispersion and dissipation properties of the WENO-ZA scheme}

In this section, the dispersion and dissipation properties of the present scheme are investigated by analyzing its spectral properties. The representation for the approximate dispersion relation of linear and nonlinear finite-difference schemes 


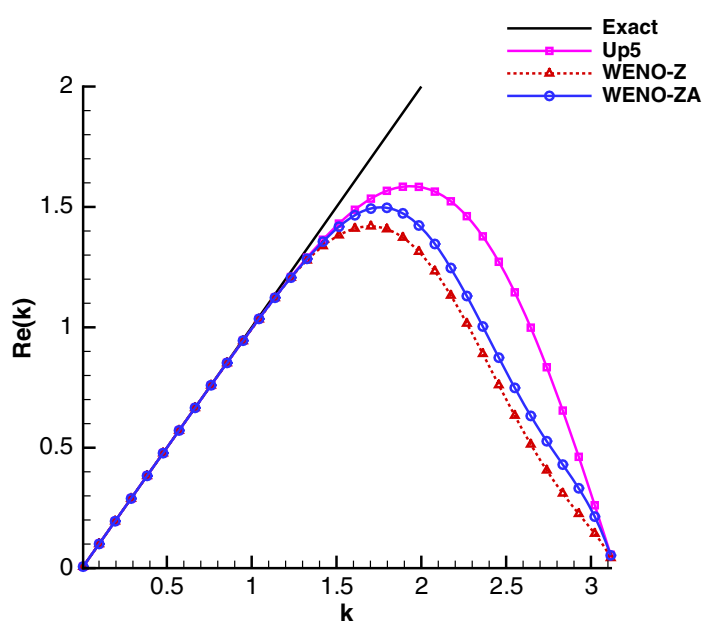

(A) Dispersion

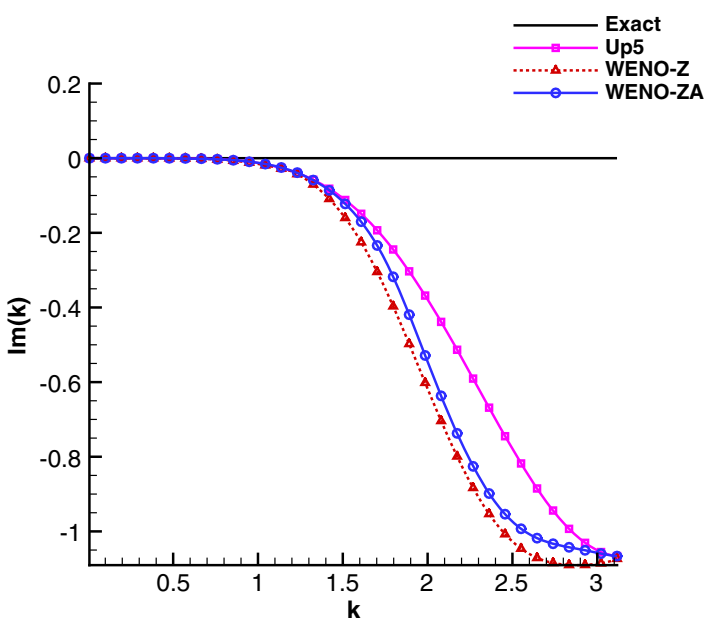

(B) Dissipation

FIGURE 1 The dispersion and dissipation properties. Up5, fifth-order upstream; WENO, weighted essentially nonoscillatory [Colour figure can be viewed at wileyonlinelibrary.com]

can be found in the work of Pirozzoli. ${ }^{15}$ As shown in Figure 1, compared with the WENO-Z scheme, the present scheme improves both the dispersion and dissipation errors effectively.

\section{4 | NUMERICAL EXAMPLES}

In this section, several linear advection problems, ie, 1- and 2-dimensional Euler problems are calculated, and in order to show the good performance of the new scheme, the following schemes are compared:

1. WENO-Z ${ }^{7}$ : GSI $\tau_{5}(14)$ and $q=1$;

2. WENO-Z2 ${ }^{7}$ : GSI $\tau_{5}(14)$ and $q=2$;

3. WENO-NS ${ }^{9}$ : GSI $\zeta(17)$ and $\xi=0.1$ for linear problems, $\xi=0.4$ for nonlinear problems;

4. WENO-P ${ }^{10}$ : GSI $\zeta(18)$ and $\delta=0.05$;

5. WENO-Z $\eta^{11}$ : GSIs are $\tau_{6}(20), \tau_{81}$, and $\tau_{82}(21)$, respectively, and $q=1$;

6. WENO-Z $+{ }^{13}$ : weighting method (22) and GSI $\tau_{5}$ (14), and $\lambda=\Delta x^{2 / 3}$;

7. WENO-Z $\tau_{6}^{\prime}$ : GSI $\tau_{6}^{\prime}(24)$ and $q=1$;

8. WENO-Z $\tau_{6}^{\prime} 2$ : GSI $\tau_{6}^{\prime}(24)$ and $q=2$;

9. WENO-ZA: weighting method (25) and GSI $\tau_{6}^{\prime}(24)$.

For convenience, the purpose of these comparisons is outlined as follows.

1. Testing WENO-Z $\tau_{6}^{\prime}$ (see Table 1 and Figure 8 ) is to show that, comparing to other GSIs, $\tau_{6}^{\prime}$ is a compromise GSI, which can keep the ENO property and achieve high-order accuracy simultaneously.

2. Testing WENO-Z2 and WENO- $\tau_{6}^{\prime} 2$ (see Table 1 and Figures 4 and 8 ) is to show that, comparing the power $q=1$, $q=2$ can improve the accuracy order for a smooth solution, but it really increases the numerical dissipation near discontinuities.

3. The comparisons of WENO- $\tau_{6}^{\prime}$, WENO- $\tau_{6}^{\prime} 2$, and WENO-ZA (see Table 1 and Figures 4 and 9 ) are used to show that the new weighting method (25) is necessary and valuable for both improving the accuracy in smooth regions and decreasing the dissipation near discontinuities.

4. The comparisons of WENO-ZA, WENO-Z, WENO-Z $\eta$, WENO-NS, and WENO-P (see Sections 4.1-4.3) are used to show the comprehensive advantages of WENO-ZA over the others, including ENO property, high-order accuracy, high resolution, and low dissipation.

In this paper, time advancement is performed with the fourth-order Runge-Kutta method. ${ }^{16}$ 
TABLE 1 Convergence order at the critical point

\begin{tabular}{|c|c|c|c|c|c|c|c|c|}
\hline \multirow{3}{*}{$\frac{\Delta x}{5.00 E-3}$} & \multirow{2}{*}{\multicolumn{2}{|c|}{$\begin{array}{ll}\text { Error } & \text { Order } \\
\text { Up5 } & \end{array}$}} & \multirow{2}{*}{\multicolumn{2}{|c|}{$\begin{array}{c}\text { Error Order } \\
\text { WENO-JS }\end{array}$}} & \multirow{2}{*}{\multicolumn{2}{|c|}{$\begin{array}{l}\text { Error Order } \\
\text { WENO-Z }\end{array}$}} & \multirow{2}{*}{\multicolumn{2}{|c|}{$\begin{array}{l}\text { Error Order } \\
\text { WENO-Z2 }\end{array}$}} \\
\hline & & & & & & & & \\
\hline & $5.208 \mathrm{E}-14$ & - & $1.566 \mathrm{E}-07$ & - & 4.133E-09 & - & $1.118 \mathrm{E}-10$ & - \\
\hline $2.50 \mathrm{E}-3$ & $1.628 \mathrm{E}-15$ & 5.000 & $2.323 \mathrm{E}-08$ & 2.752 & $3.353 \mathrm{E}-10$ & 3.624 & $1.128 \mathrm{E}-11$ & 3.309 \\
\hline $1.25 \mathrm{E}-3$ & $5.086 \mathrm{E}-17$ & 5.000 & $3.138 \mathrm{E}-09$ & 2.888 & $2.357 \mathrm{E}-11$ & 3.831 & $5.866 \mathrm{E}-13$ & 4.266 \\
\hline $6.25 \mathrm{E}-4$ & $1.589 \mathrm{E}-18$ & 5.000 & $4.070 \mathrm{E}-10$ & 2.947 & $1.558 \mathrm{E}-12$ & 3.919 & $2.200 \mathrm{E}-14$ & 4.737 \\
\hline $3.12 \mathrm{E}-4$ & $4.967 \mathrm{E}-20$ & 5.000 & $5.180 \mathrm{E}-11$ & 2.974 & $1.001 \mathrm{E}-13$ & 3.960 & $7.448 \mathrm{E}-16$ & 4.884 \\
\hline$\Delta x$ & \multicolumn{2}{|c|}{ WENO-NS } & \multicolumn{2}{|c|}{ WENO-P } & \multicolumn{2}{|c|}{ WENO-Z $\eta\left(\tau_{6}\right)$} & \multicolumn{2}{|c|}{ WENO-Z $\eta\left(\tau_{81}\right)$} \\
\hline $5.00 \mathrm{E}-3$ & $5.266 \mathrm{E}-11$ & - & $8.233 \mathrm{E}-10$ & - & $1.142 \mathrm{E}-11$ & - & $5.209 \mathrm{E}-14$ & - \\
\hline $2.50 \mathrm{E}-3$ & $1.938 \mathrm{E}-12$ & 4.764 & $4.776 \mathrm{E}-11$ & 4.108 & $4.756 \mathrm{E}-13$ & 4.586 & $1.628 \mathrm{E}-15$ & 5.000 \\
\hline $1.25 \mathrm{E}-3$ & $6.472 \mathrm{E}-14$ & 4.905 & $2.892 \mathrm{E}-12$ & 4.046 & $1.672 \mathrm{E}-14$ & 4.830 & $5.086 \mathrm{E}-17$ & 5.000 \\
\hline $6.25 \mathrm{E}-3$ & $2.084 \mathrm{E}-15$ & 4.957 & $1.782 \mathrm{E}-13$ & 4.021 & $5.518 \mathrm{E}-16$ & 4.922 & $1.589 \mathrm{E}-18$ & 5.000 \\
\hline $3.12 \mathrm{E}-3$ & $6.604 \mathrm{E}-17$ & 4.980 & $1.106 \mathrm{E}-14$ & 4.010 & $1.770 \mathrm{E}-17$ & 4.962 & 4.967E-20 & 5.000 \\
\hline$\Delta x$ & \multicolumn{2}{|c|}{ WENO-Z+ } & \multicolumn{2}{|c|}{ WENO-Z $\tau_{6}^{\prime}$} & \multicolumn{2}{|c|}{ WENO-Z $\tau_{6}^{\prime} 2$} & \multicolumn{2}{|c|}{ WENO-ZA } \\
\hline $5.00 \mathrm{E}-3$ & $3.417 \mathrm{E}-08$ & - & $1.217 \mathrm{E}-10$ & - & $1.039 \mathrm{E}-13$ & - & $9.634 \mathrm{E}-14$ & - \\
\hline $2.50 \mathrm{E}-3$ & $4.975 \mathrm{E}-09$ & 2.780 & $5.059 \mathrm{E}-12$ & 4.589 & $3.979 \mathrm{E}-15$ & 4.706 & $2.539 \mathrm{E}-15$ & 5.246 \\
\hline $1.25 \mathrm{E}-3$ & $7.174 \mathrm{E}-10$ & 2.794 & $1.778 \mathrm{E}-13$ & 4.831 & $8.315 \mathrm{E}-17$ & 5.581 & $6.019 \mathrm{E}-17$ & 5.398 \\
\hline $6.25 \mathrm{E}-3$ & $1.019 \mathrm{E}-10$ & 2.816 & $5.863 \mathrm{E}-15$ & 4.922 & $1.896 \mathrm{E}-18$ & 5.455 & $1.671 \mathrm{E}-18$ & 5.171 \\
\hline $3.12 \mathrm{E}-3$ & $1.427 \mathrm{E}-11$ & 2.836 & $1.880 \mathrm{E}-16$ & 4.963 & $5.228 \mathrm{E}-20$ & 5.181 & $5.034 \mathrm{E}-20$ & 5.053 \\
\hline
\end{tabular}

Abbreviations: Up5, fifth-order upstream; WENO, weighted essentially nonoscillatory.

\section{1 | The convergence rate at critical point}

The function $f(x)=x^{3}+\cos (x)$ is used to test the numerical convergence order of different schemes. At $x=0$, there is $f_{x=0}^{\prime}=$ 0 and $f_{x=0}^{\prime \prime \prime} \neq 0$. As shown in Table 1 , the WENO-Z scheme and WENO-P scheme only get fourth-order accuracy. The WENO-Z2, WENO-NS, WENO-Z $\eta\left(\tau_{6}\right)$, WENO-Z $\eta\left(\tau_{81}\right)$, WENO-Z $\tau_{6}^{\prime}$, WENO-Z $\tau_{6}^{\prime} 2$, and WENO-ZA schemes get fifth-order accuracy, but only the WENO-Z $\eta\left(\tau_{81}\right)$, WENO-Z $\tau_{6}^{\prime} 2$, and WENO-ZA schemes can obtain the errors with the same order of magnitude as those of the fifth-order upstream (Up5) scheme, and the errors generated by the other schemes are at least 3 orders of magnitude larger than those of the WENO-ZA scheme. WENO-Z $\eta\left(\tau_{82}\right)$ has similar results as WENO-Z $\eta\left(\tau_{81}\right)$ and hence is not shown in this Table.

It should be noted that, for this case, the WENO-Z+ scheme can only obtain third-order accuracy, as well as the WENO-JS scheme. In addition, since the parameter $\lambda$ is dependent on the grid spacing, the resulting WENO-Z+ scheme no longer preserves the scale invariance. This shortcoming greatly limits the applications of WENO-Z+. For the WENO-P scheme, the parameter $\delta$ in (19) generates different coefficients in different $I S_{k}$; hence, the accuracy rate is influenced.

As the results of the WENO-Z $\tau_{6}^{\prime}$ and WENO-Z $\tau_{6}^{\prime} 2$ schemes have shown, WENO-Z $\tau_{6}^{\prime}$ obtains similar results as the WENO-Z2 scheme since, at critical point, there are $\tau_{6}^{\prime} /\left(I S_{k}+\epsilon\right)=O\left(\Delta x^{2}\right)$ and $\left[\tau_{5} /\left(I S_{k}+\epsilon\right)\right]^{2}=O\left(\Delta x^{2}\right)$. In addition, as analyzed in Section 3.2, for the smooth solution, the WENO-Z $\tau_{6}^{\prime} 2$ scheme obtains almost the same errors as the new scheme with function $A$ (WENO-ZA).

\section{2 | Linear advection problems}

In this section, the linear advection Equation (37) is solved to compare the shock-capturing ability, robustness, and low-dissipation property of different schemes.

$$
\begin{cases}\frac{\partial u}{\partial t}+\frac{\partial u}{\partial x}=0, & x_{0} \leqslant x \leqslant x_{1}, \\ u(x, t=0)=u_{0}(x), & \text { periodic boundary }\end{cases}
$$

The exact solution of (37) is $u(x, t)=u_{0}(x-t)$.

\subsection{1 | Case 1: Linear problem with a discontinuity}

$$
u_{0}(x)= \begin{cases}-\sin (\pi x)-\frac{1}{2} x^{3}, & -1 \leq x<0, \\ -\sin (\pi x)-\frac{1}{2} x^{3}+1, & 0 \leq x \leq 1 .\end{cases}
$$




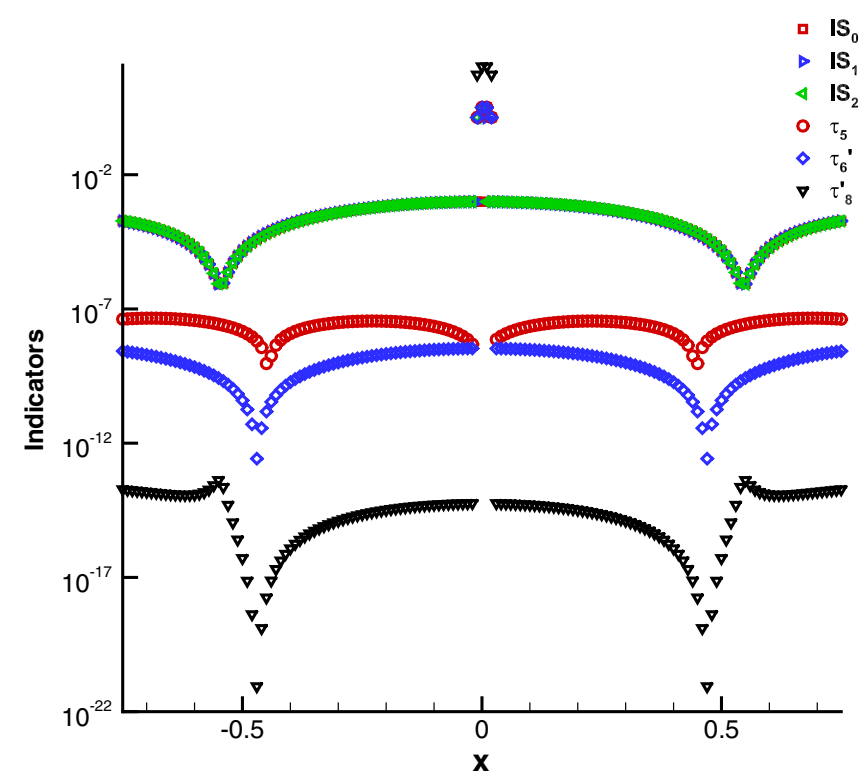

FIGURE 2 Distribution of LSIs and GSIs at $t=0$ [Colour figure can be viewed at wileyonlinelibrary.com]

First, the solution, which contains a discontinuity at $x=0$, is used to show the behavior of the new GSI $\tau_{8}^{\prime}=A \cdot \tau_{6}^{\prime}(36)$ in the smooth regions and discontinuous regions. Figure 2 is the distribution of the LSIs $I S_{k}$ and 3 GSIs, ie, $\tau_{5}, \tau_{6}^{\prime}$, and $\tau_{8}^{\prime}$, for the initial solution. As shown in Figure 2, the value of $\tau_{8}^{\prime}$ is the smallest one in smooth regions, which indicates that $\tau_{8}^{\prime}$ is an infinitesimal with higher order than $\tau_{5}$ and $\tau_{6}^{\prime}$; hence, the new scheme is more accurate than WENO-Z. Although $\tau_{8}^{\prime}$ is larger than the others near discontinuity, it does not break the ENO property, as illustrated in (32). In addition, Figure 3 shows that, near discontinuity, the weights of the new scheme are almost the same as those of WENO-Z; both of them assign larger weights to substencils containing discontinuity than WENO-JS (for example, see $\omega_{2}^{J S}, \omega_{2}^{Z}$, and $\omega_{2}^{Z A}$ ). Hence, as Borges et $\mathrm{al}^{7}$ pointed out, for the solution containing discontinuities, WENO-Z is less dissipative than WENO-JS because of the relative large weights assigned to the substencils containing discontinuities, and the same holds for the present scheme.

The numerical results at $t=2$ with $N=200$ and $\Delta t=\Delta x / 2$ are plotted in Figure 4. Near discontinuity, the new scheme shows less dissipation than the WENO-Z $\tau_{6}^{\prime} 2$ scheme and it is closer to the WENO-Z $\tau_{6}^{\prime}$. This also indicates that the power $q=2$ brings larger dissipation than $q=1$, and the present weighting method (WENO-ZA) is effective in decreasing dissipation near discontinuities.

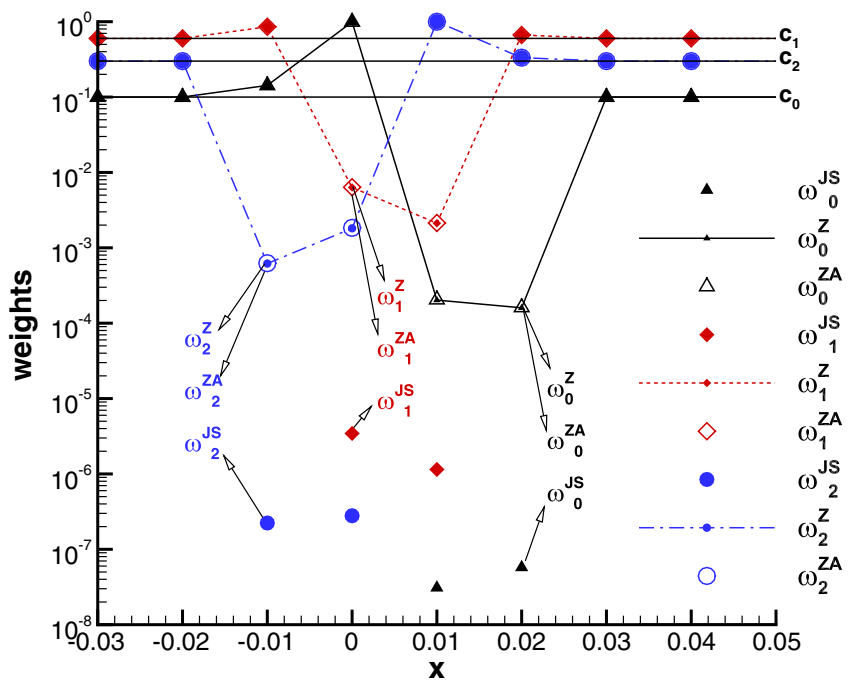

FIGURE 3 Distribution of weights near discontinuity [Colour figure can be viewed at wileyonlinelibrary.com] 


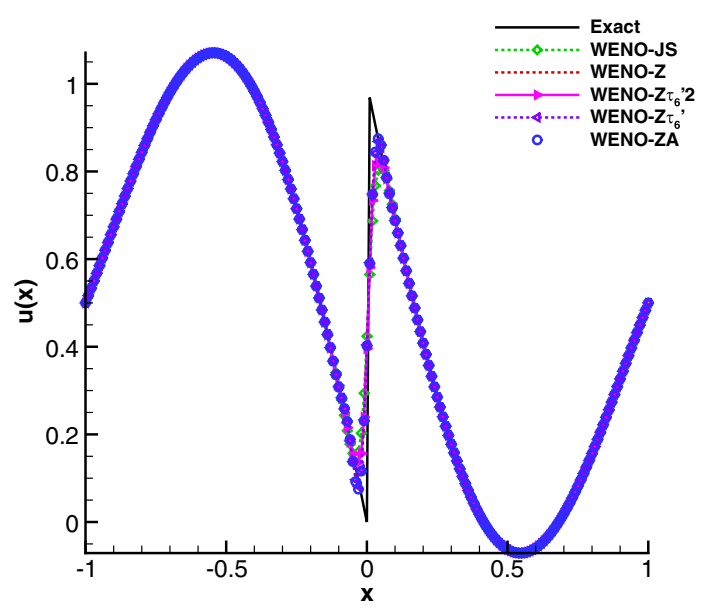

(A)

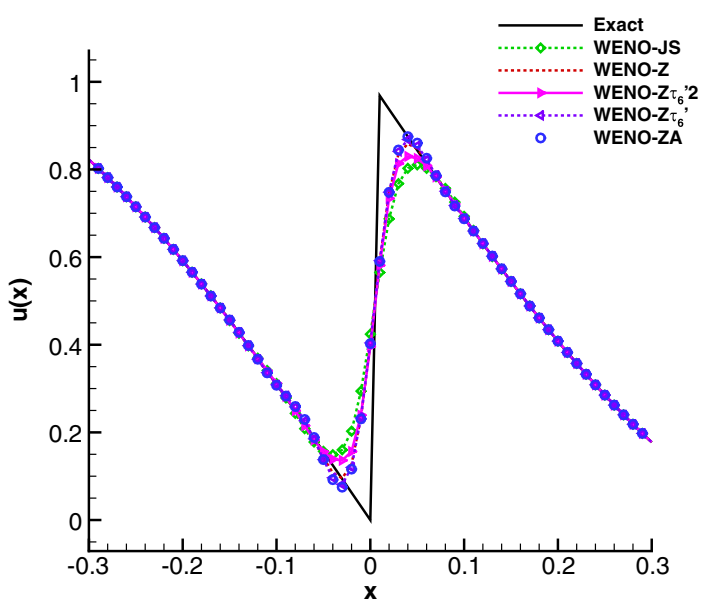

(B)

FIGURE 4 Case 1: $t=2$ and $N=200$, using WENO-JS, WENO-Z, WENO-Z $\tau_{6}^{\prime} 2$, and WENO-ZA. WENO, weighted essentially nonoscillatory [Colour figure can be viewed at wileyonlinelibrary.com]

TABLE $2 L_{\infty}$ errors, orders, and CPU times of Case 2 at $t=2$

\begin{tabular}{cccccccccc} 
N & \multicolumn{3}{c}{ Up5 } & \multicolumn{2}{c}{ WENO-Z } & \multicolumn{2}{c}{ WENO-ZA } \\
& Error & Order & Time (Ratio) & Error & Order & Time (Ratio) & Error & Order & Time (Ratio) \\
\hline 80 & $6.701 \mathrm{E}-06$ & - & $1.404 \mathrm{E}-01(0.31)$ & $6.677 \mathrm{E}-06$ & - & $4.524 \mathrm{E}-01(1)$ & $6.701 \mathrm{E}-06$ & - & $4.680 \mathrm{E}-01(1.03)$ \\
160 & $2.099 \mathrm{E}-07$ & 5.00 & $7.176 \mathrm{E}-01(0.35)$ & $2.099 \mathrm{E}-07$ & 4.99 & $2.075 \mathrm{E}+00(1)$ & $2.099 \mathrm{E}-07$ & 5.00 & $2.293 \mathrm{E}+00(1.11)$ \\
320 & $6.553 \mathrm{E}-09$ & 5.00 & $2.668 \mathrm{E}+00(0.29)$ & $7.767 \mathrm{E}-09$ & 4.76 & $9.282 \mathrm{E}+00(1)$ & $6.552 \mathrm{E}-09$ & 5.00 & $1.025 \mathrm{E}+01(1.10)$ \\
640 & $2.046 \mathrm{E}-10$ & 5.00 & $1.260 \mathrm{E}+01(0.30)$ & $3.570 \mathrm{E}-10$ & 4.44 & $4.256 \mathrm{E}+01(1)$ & $2.046 \mathrm{E}-10$ & 5.00 & $4.699 \mathrm{E}+01(1.10)$ \\
1280 & $6.392 \mathrm{E}-12$ & 5.00 & $6.004 \mathrm{E}+01(0.30)$ & $1.725 \mathrm{E}-11$ & 4.37 & $2.013 \mathrm{E}+02(1)$ & $6.392 \mathrm{E}-12$ & 5.00 & $2.223 \mathrm{E}+02(1.10)$ \\
\hline
\end{tabular}

Abbreviations: Up5, fifth-order upstream; WENO, weighted essentially nonoscillatory.

\subsection{2 | Case 2: Linear problem to test the accuracy}

$$
u_{0}(x)=\sin \left(\pi x-\frac{\sin (\pi x)}{\pi}\right),-1 \leq x \leq 1
$$

This solution ${ }^{6}$ is often used to test the convergence order in $L_{\infty}$ norm of WENO schemes. The errors, orders, and CPU time of the Up5, WENO-Z, and WENO-ZA schemes are compared in Table 2. The time step $\Delta t=\Delta x^{5 / 4}$ is chosen, and the Fortran 95 program with quadruple precision is performed on Intel Xeon E5-2609 V4. As shown in this table, the WENO-ZA scheme gets fifth-order accuracy as the Up5 scheme does, but the WENO-Z scheme fails. Figure 5 gives the comparisons of computational efficiency. It can be seen that WENO-ZA costs about $10 \%$ CPU time more than WENO-Z; however, if high accuracy (for example, $L_{\infty}<1 \times 10^{-8}$ for this case) is required, the new scheme is more efficient than the WENO-Z scheme.

\subsection{3 | Case 3: Linear problem with several critical points}

$$
u_{0}(x)=e^{-(x-90)^{2} / 400}\left(\cos \left(\frac{\pi}{8}(x-90)\right)+\cos \left(\frac{\pi}{4}(x-90)\right)\right), 50 \leq x \leq 130
$$

This smooth solution containing several critical points is used to test the accuracy and numerical dissipation of different schemes. The numerical solutions at $t=400$ are calculated with $N=100$ and $\Delta t=\Delta x / 2$. As shown in Figure 6, the WENO-ZA scheme obtains almost the same results as Up5; it behaves much better than WENO-Z. The results computed by the WENO-NS, WENO-P, and WENO-Z $\eta$ schemes are also shown in Figure 7. The results of the WENO-Z $\eta$ schemes (with high-order GSIs $\tau_{6}, \tau_{81}$, and $\tau_{82}$ ) are closer to that of WENO-ZA. However, due to the unbalanced contribution provided by 3 substencils in the WENO-NS and WENO-P schemes, ${ }^{10}$ both of them generate other issues such as overestimated extremum and obvious phase error. 


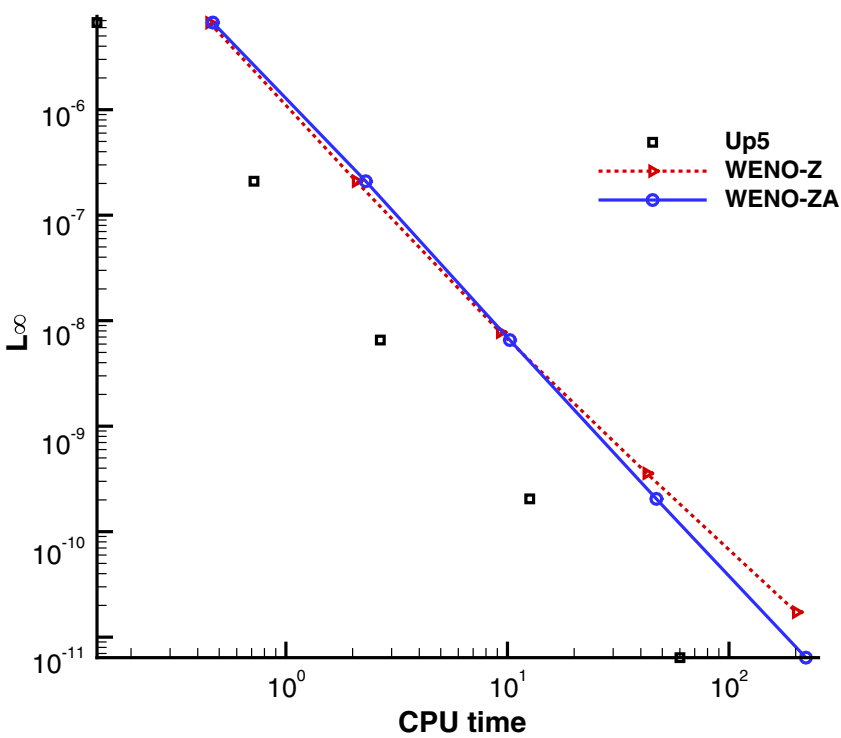

FIGURE 5 Comparison of the computational efficiency. Up5, fifth-order upstream; WENO, weighted essentially nonoscillatory [Colour figure can be viewed at wileyonlinelibrary.com]

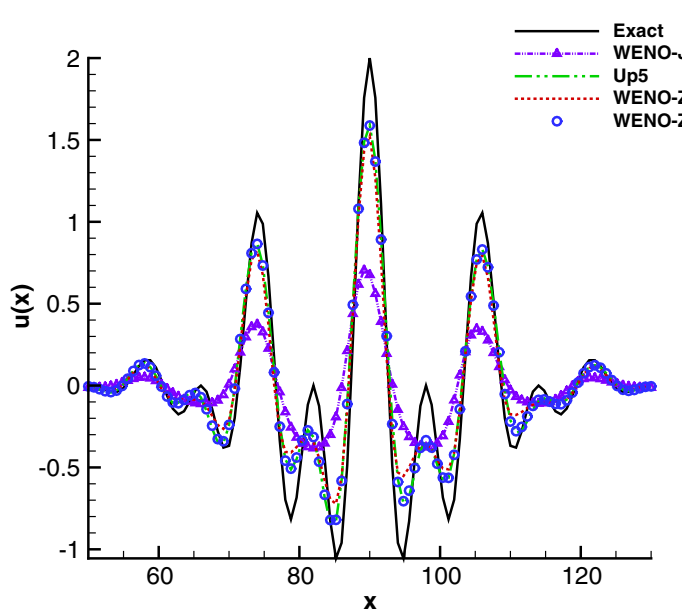

(A)

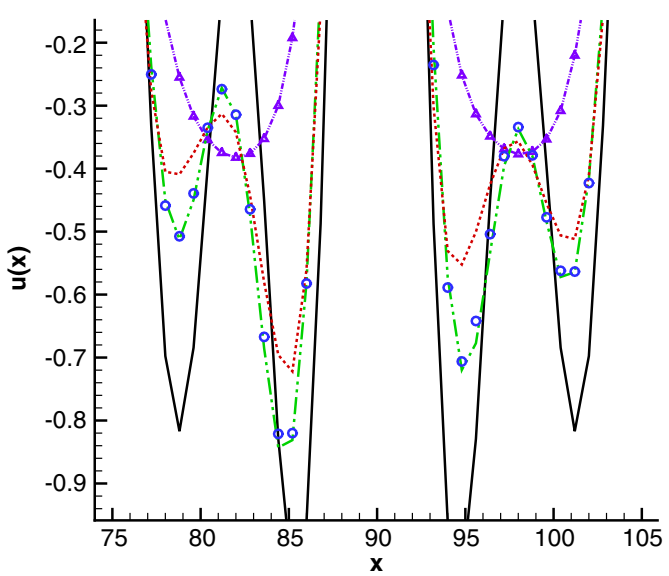

(B)

FIGURE 6 Case 3: $t=400$ and $N=100$, using WENO-JS, WENO-Z, Up5, and WENO-ZA. Up5, fifth-order upstream; WENO, weighted essentially nonoscillatory [Colour figure can be viewed at wileyonlinelibrary.com]

\subsection{4 | Case 4: Combine-wave problem}

$$
\begin{gathered}
u(x, 0)= \begin{cases}\frac{1}{6}(G(x, \beta, z-\delta)+G(x, \beta, z+\delta)+4 G(x, \beta, z)), & -0.8 \leqslant x<-0.6, \\
1, & -0.4 \leqslant x<-0.2, \\
1-|10(x-0.1)|, & 0 \leqslant x<0.2, \\
\frac{1}{6}(F(x, \alpha, a-\delta)+F(x, \alpha, a+\delta)+4 F(x, \alpha, a)), & 0.4 \leqslant x<0.6, \\
0, & \text { otherwise }\end{cases} \\
G(x, \beta, z)=e^{-\beta(x-z)^{2}}, F(x, \alpha, a)=\sqrt{\max \left(1-\alpha^{2}(x-a)^{2}, 0\right)}
\end{gathered}
$$

The solution consisting of a Gaussian, a triangle, a square wave, and a semiellipse wave is calculated to validate the robustness of different schemes. The constants are $z=-0.7, \delta=0.005, \beta=\ln (2) /\left(36 \delta^{2}\right), a=0.5$, and $\alpha=10$. It is solved until $t=10$ with $N=200$ and $\Delta t=\Delta x / 2$. 


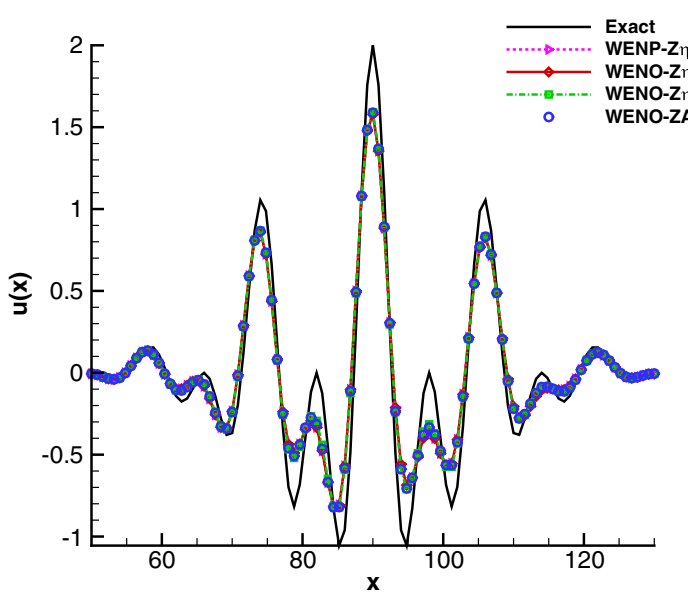

(A)

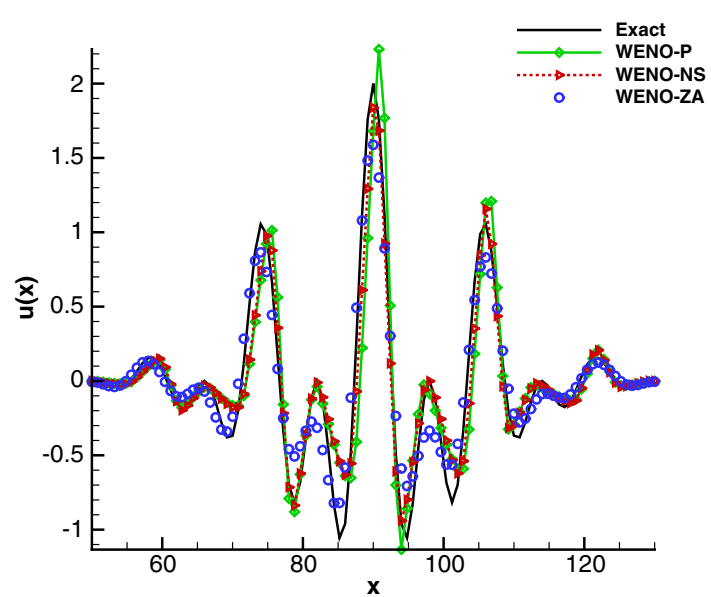

(B)

FIGURE 7 Case 3: $t=400$ and $N=100$, using WENO-Z $\eta$, WENO-NS, WENO-P, and WENO-ZA. WENO, weighted essentially nonoscillatory [Colour figure can be viewed at wileyonlinelibrary.com]

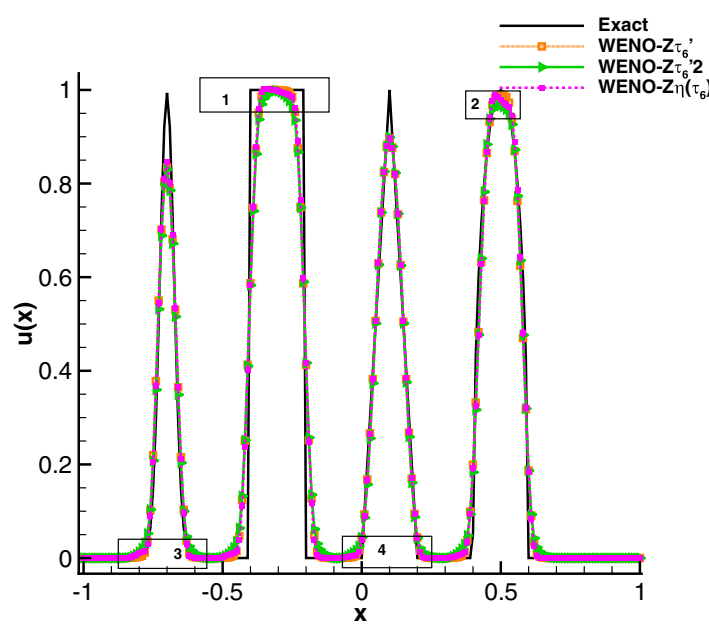

(A)

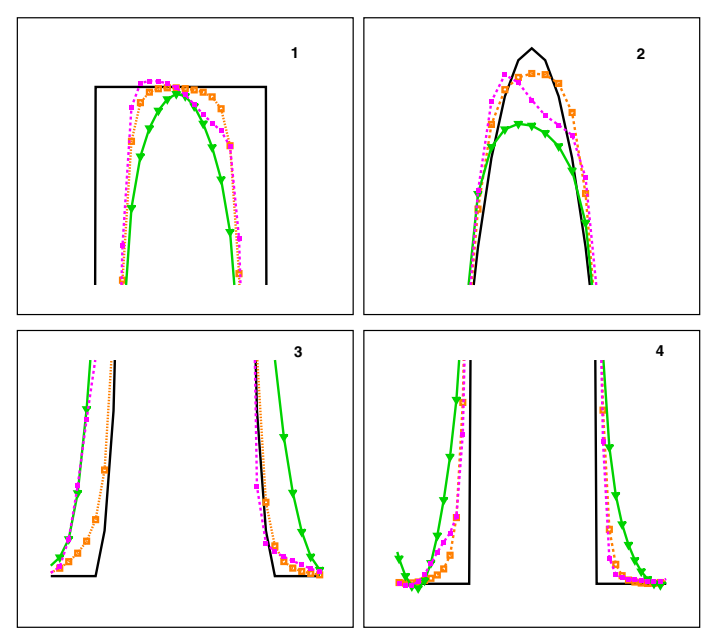

(B)

FIGURE 8 Case 4: $t=10$ and $N=200$, using WENO-Z $\eta\left(\tau_{6}\right)$, WENO-Z $\tau_{6}^{\prime}$, and WENO-Z $\tau_{6}^{\prime} 2$. WENO, weighted essentially nonoscillatory [Colour figure can be viewed at wileyonlinelibrary.com]

The results of the WENO-Z $\tau_{6}^{\prime}$, WENO-Z $\tau_{6}^{\prime} 2$, and WENO-Z $\eta\left(\tau_{6}\right)$ are compared in Figure 8 . As shown in previous cases, the power $q=2$ (WENO-Z $\tau_{6}^{\prime}{ }^{2}$ ) generates larger dissipation than $q=1$ (WENO-Z $\tau_{6}^{\prime}$ ). The GSI $\tau_{6}\left(\mathrm{WENO}-\mathrm{Z} \tau_{6}{ }^{11}\right.$ ) obtains clear overestimated and dissymmetric solutions.

The comparisons of WENO-Z, WENO-Z $\tau_{6}^{\prime}$, and WENO-ZA are given in Figure 9. From the enlarged parts (Figure 9B), it can be seen that WENO-ZA shows less dissipation than the other 2 schemes.

The results of the WENO-Z $\eta$, WENO-NS, and WENO-P schemes are also plotted in Figures 10 and 11 . As we can see the WENO-Z $\eta$ schemes (with higher-order GSIs $\tau_{81}$ and $\tau_{82}$ ) prone to generate oscillatory solutions near the discontinuities, the WENO-NS and WENO-P scheme obtain overshoot Gaussian wave and dissymmetric semiellipse wave and they also generate larger dissipation for the square wave than WENO-ZA.

From the comparisons of aforementioned cases, we can conclude that

1. $\tau_{6}^{\prime}(24)$ proposed in this paper is a compromise GSI, which can keep the ENO property and achieve high-order accuracy simultaneously;

2. The new weighting method (25) is necessary and valuable for both improving the accuracy in smooth regions and decreasing the dissipation near discontinuities. 


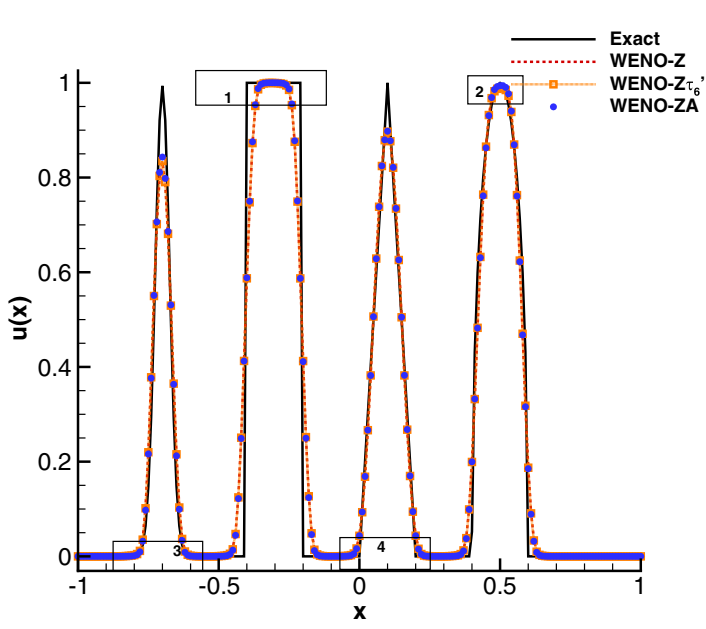

(A)

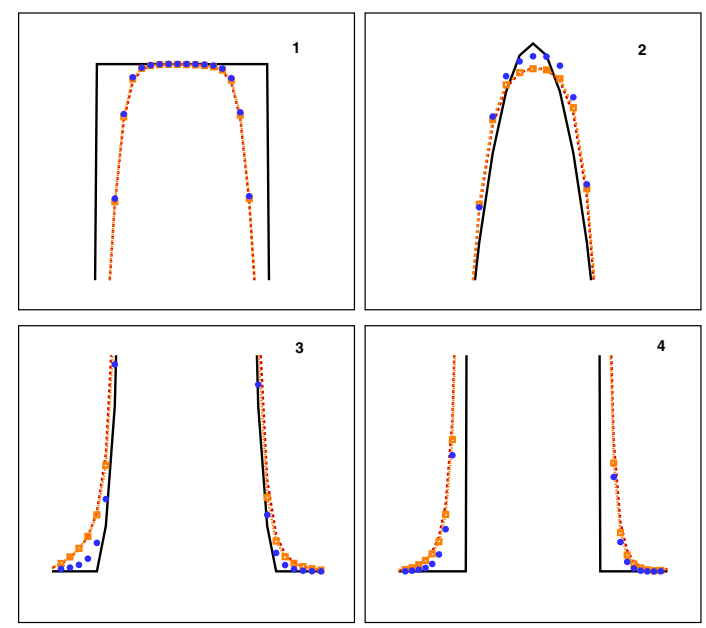

(B)

FIGURE 9 Case 4: $t=10$ and $N=200$, using WENO-Z, WENO-Z $\tau_{6}^{\prime}$, and WENO-ZA. WENO, weighted essentially nonoscillatory [Colour figure can be viewed at wileyonlinelibrary.com]

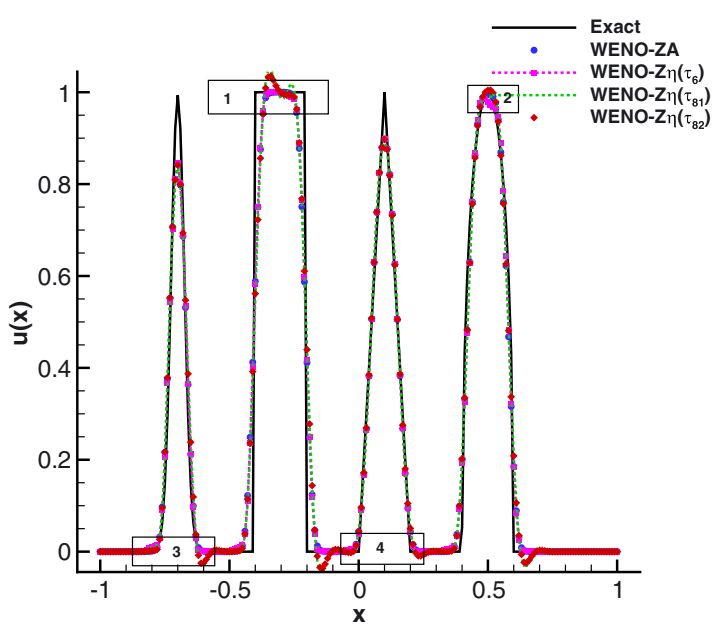

(A)

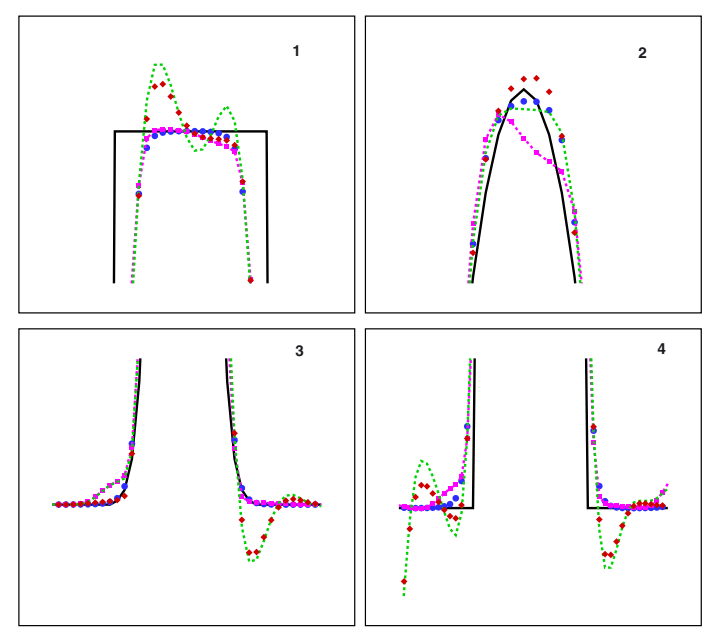

(B)

FIGURE 10 Case 4: $t=10$ and $N=200$, using WENO-Z $\eta$ and WENO-ZA. WENO, weighted essentially nonoscillatory [Colour figure can be viewed at wileyonlinelibrary.com]

\section{3 | One-dimensional Euler problems}

Next, we further study the behaviors of the WENO-ZA scheme in the 1-dimensional Euler problems. The governing equations are as follows:

$$
\frac{\partial U}{\partial t}+\frac{\partial F}{\partial x}=0
$$

where $U=(\rho, \rho u, E)^{T}, F(U)=\left(\rho u, \rho u^{2}+p, u(E+p)\right)^{T} . \rho, u, E$, and $p$ are density, velocity, total energy, and pressure, respectively, and for ideal gas $E=\frac{p}{\gamma-1}+\frac{1}{2} \rho u^{2}, \gamma=1.4$ is the ratio of specific heat. Time step is taken as

$$
\Delta t=\frac{\sigma \Delta x}{\max _{i}\left(\left|u_{i}\right|+c_{i}\right)},
$$

where $\sigma=0.5$ is CFL number. $c$ is the speed of sound and given by $c=\sqrt{\gamma p / \rho}$. The characteristicwise Roe-type decomposition method ${ }^{17}$ is used for the local characteristic reconstruction. All the reference solutions were obtained by the WENO-Z scheme with a grid of 2000. 


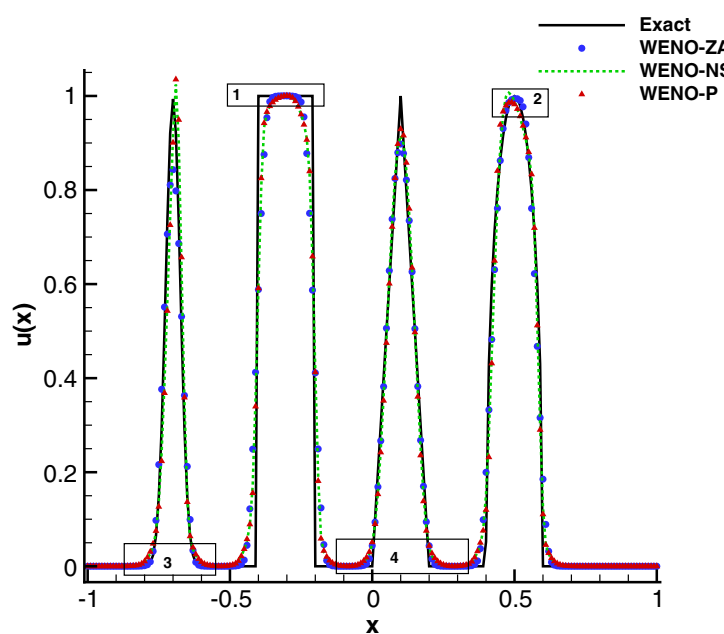

(A)

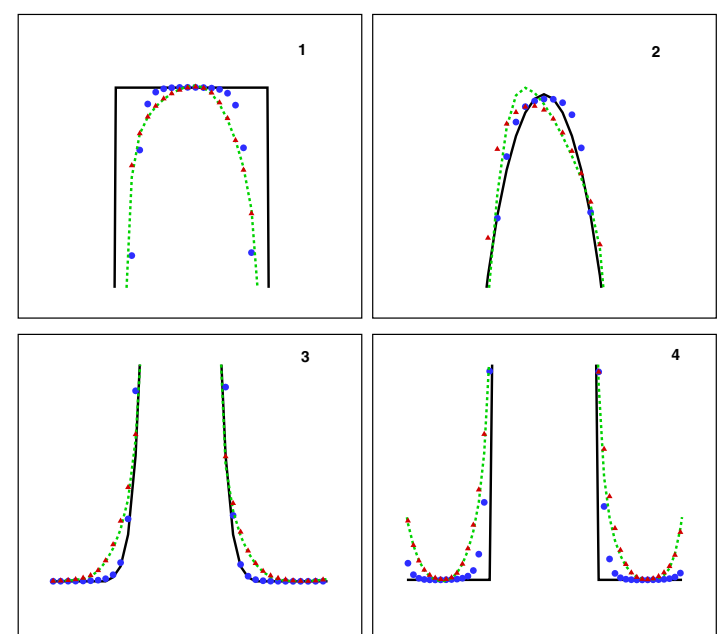

(B)

FIGURE 11 Case 4: $t=10$ and $N=200$, using WENO-NS, WENO-P, and WENO-ZA. WENO, weighted essentially nonoscillatory [Colour figure can be viewed at wileyonlinelibrary.com]

\subsection{1 | Case 5: Shu-Osher problem}

The first 1-dimensional case is the Shu-Osher problem ${ }^{16}$ with the initial conditions

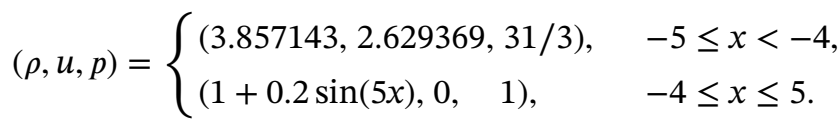

It is often used as a benchmark case to test the performance of different schemes. Figures 12-14 give the distributions of density at $t=1.8$ with $N=200$. As these figures have shown, the WENO-ZA scheme is less dissipative than WENO-Z (Figure 12), and it is also comparable with those improved schemes (such as WENO-Z $\eta$, WENO-NS, and WENO-P). However, although WENO-Z $\eta$, WENO-NS, and WENO-P can resolve this kind of high-frequency wave structure well, their dissipation is not large enough to suppress the oscillations in some problems with a strong shock, for example, they even cannot finish the calculation of the blast-waves interaction problem in the next case. Applications to the current case and the next case show the conclusion again, ie, the WENO-ZA scheme proposed in this paper can keep the ENO property and has high accuracy and low dissipation, simultaneously.

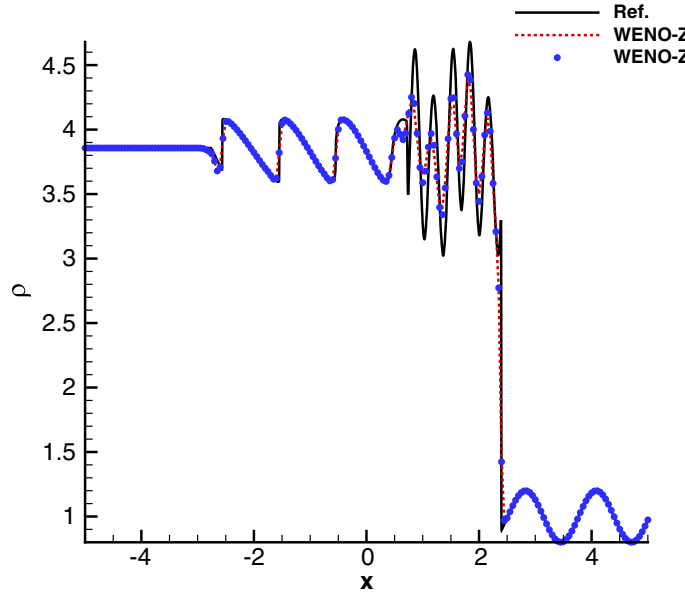

(A)

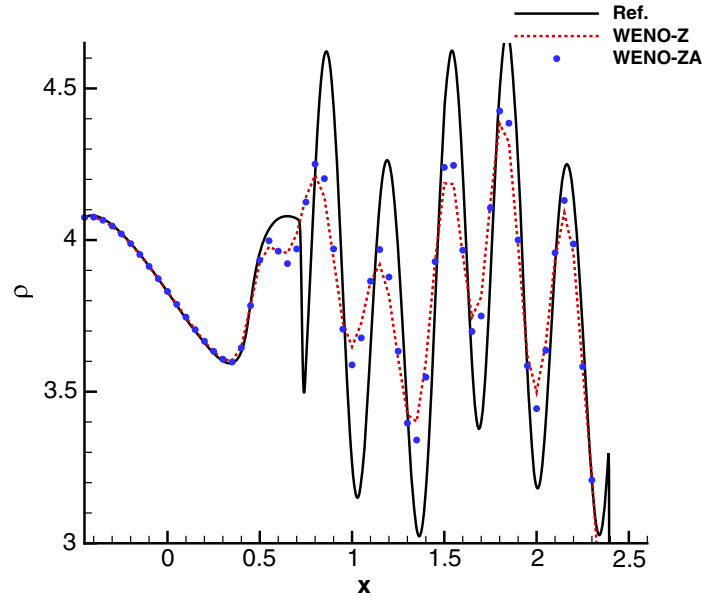

(B)

FIGURE 12 Shu-Osher problem: $t=1.8$ and $N=200$, using WENO-Z and WENO-ZA. WENO, weighted essentially nonoscillatory [Colour figure can be viewed at wileyonlinelibrary.com] 


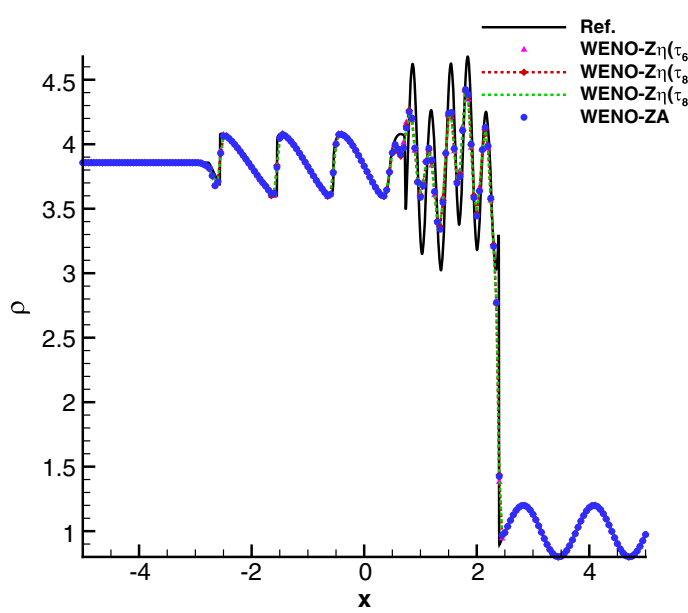

(A)

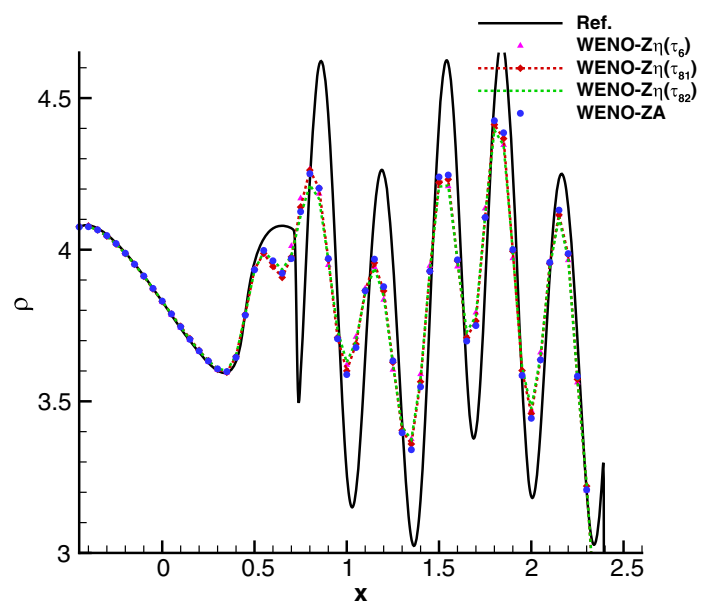

(B)

FIGURE 13 Shu-Osher problem: $t=1.8$ and $N=200$, using WENO-Z $\eta$ and WENO-ZA. WENO, weighted essentially nonoscillatory [Colour figure can be viewed at wileyonlinelibrary.com]

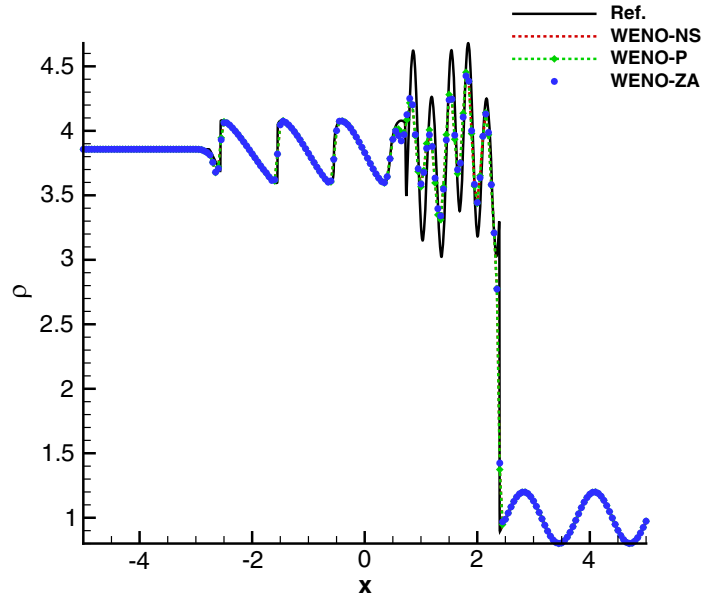

(A)

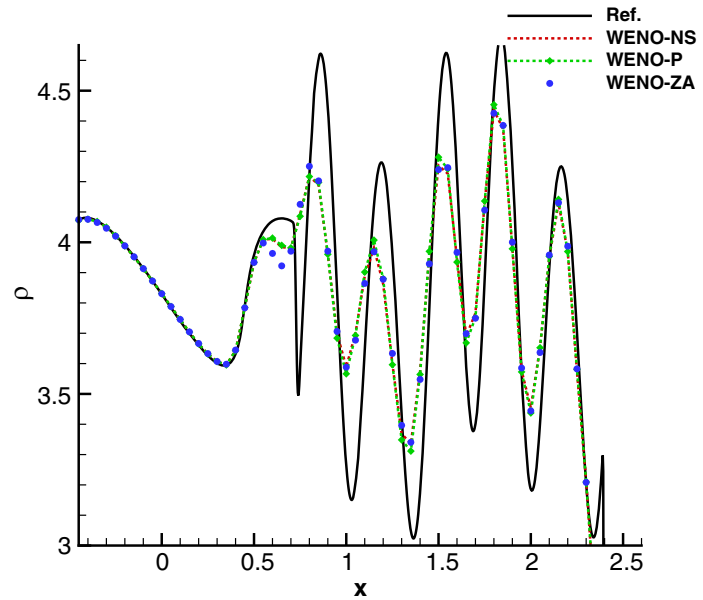

(B)

FIGURE 14 Shu-Osher problem: $t=1.8$ and $N=200$, using WENO-NS, WENO-P, and WENO-ZA. WENO, weighted essentially nonoscillatory [Colour figure can be viewed at wileyonlinelibrary.com]

\subsubsection{Case 6: Blast-waves interaction problem}

The second case is the blast-waves interaction problem $^{7}$ with the initial conditions

$$
(\rho, u, p)= \begin{cases}(1,0,1000), & 0 \leq x<0.1 \\ (1,0,0.01), & 0.1 \leq x<0.9 \\ (1,0,100), & 0.9 \leq x \leq 1\end{cases}
$$

The numerical results at $t=0.038$ with $N=300$ are presented in Figure 15. It can be seen that the WENO-ZA scheme obtains a more accurate solution than the WENO-Z scheme. As already pointed out previously, in this case, due to the small value (0.01) of pressure, a small overshoot may generate negative pressure; hence, the WENO-Z $\eta$ schemes and those schemes with unsuitable empirical parameters (WENO-NS and WENO-P) make the computation breakdown and no results can be obtained. Hence, the WENO-Z $\eta$, WENO-NS, and WENO-P schemes are no longer discussed and compared in the following 2-dimensional problems. 


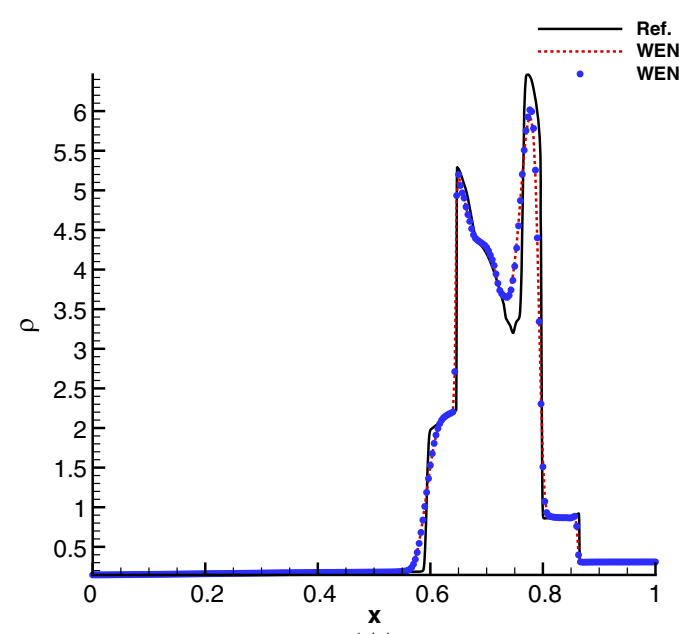

(A)

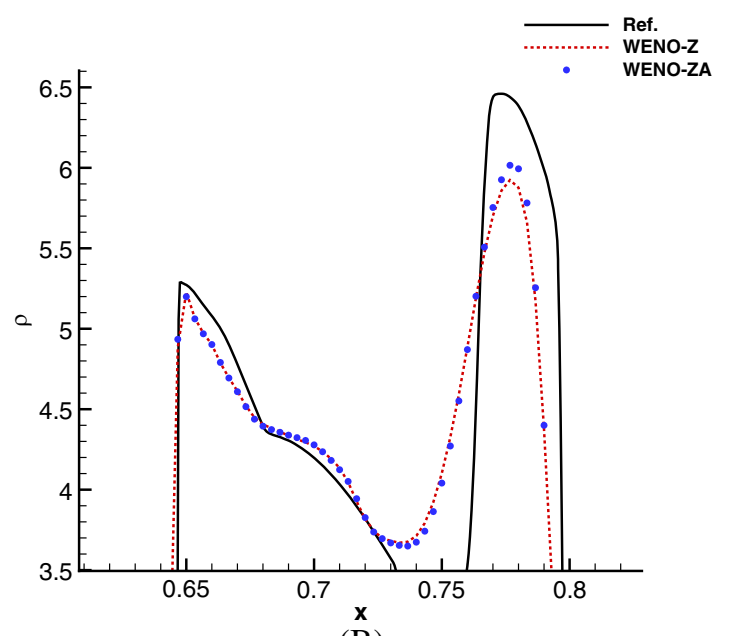

(B)

FIGURE 15 Blast-waves interactive problem: $t=0.038$ and $N=300$, using WENO-Z and WENO-ZA. WENO, weighted essentially nonoscillatory [Colour figure can be viewed at wileyonlinelibrary.com]

\section{4 | Two-dimensional Euler problems}

The governing equation is the 2-dimensional Euler equation

$$
\frac{\partial U}{\partial t}+\frac{\partial F}{\partial x}+\frac{\partial G}{\partial y}=0
$$

where the conservative variables $U$, and the inviscid flux vectors $F$ and $G$ are

$$
U=\left[\begin{array}{c}
\rho \\
\rho u \\
\rho v \\
E
\end{array}\right], \quad F=\left[\begin{array}{c}
\rho u \\
\rho u^{2}+p \\
\rho u v \\
E u+p u
\end{array}\right], \quad G=\left[\begin{array}{c}
\rho v \\
\rho u v \\
\rho v^{2}+p \\
E v+p v
\end{array}\right] .
$$

The energy is given by

$$
E=\frac{p}{\gamma-1}+\frac{\rho}{2}\left(u^{2}+v^{2}\right)
$$

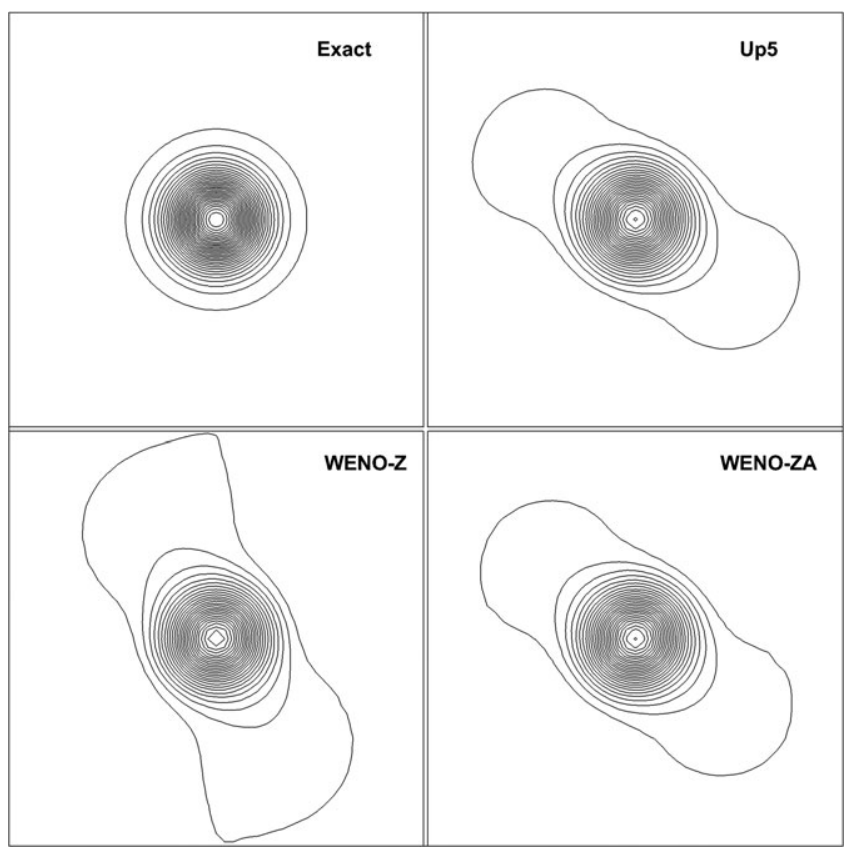

FIGURE 16 Pressure contours of the vortex propagation problem, ranging from 0.9917 to 0.9999 with 30 equally separated levels. Up5, fifth-order upstream; WENO, weighted essentially nonoscillatory 
The Roe ${ }^{18}$ flux-splitting method is used for the inviscid convective fluxes and the time step is taken as follows:

$$
\begin{aligned}
\Delta t & =\sigma \frac{\Delta t_{x} \Delta t_{y}}{\Delta t_{x}+\Delta t_{y}}, \\
\Delta t_{x} & =\frac{\Delta x}{\max _{i, j}\left(\left|u_{i, j}\right|+c_{i, j}\right)}, \\
\Delta t_{y} & =\frac{\Delta y}{\max _{i, j}\left(\left|v_{i, j}\right|+c_{i, j}\right)} .
\end{aligned}
$$

\subsection{1 | Case 7: Periodic vortex propagation problem}

This is a 2-dimensional periodic vortex propagation problem ${ }^{19,20}$ used to assess the numerical dissipation of the finite-difference schemes. The vortex is described as a perturbation to the velocity $(u, v)$, temperature $(T=p / \rho)$, and entropy $\left(S=p / \rho^{\gamma}\right)$ of the mean flow $(\rho, u, \nu, p)=(1,0.5,0,1)$,

$$
\left\{\begin{array}{l}
\hat{u}=-\frac{\epsilon e^{\left(1-r^{2}\right) / 2}}{2 \pi} \bar{y} \\
\hat{v}=\frac{\epsilon e^{\left(1-r^{2}\right) / 2}}{2 \pi} \bar{x} \\
\hat{T}=-\frac{(\gamma-1) \epsilon^{2} e^{\left(1-r^{2}\right)}}{8 \gamma \pi^{2}}, \\
\hat{S}=0
\end{array}\right.
$$

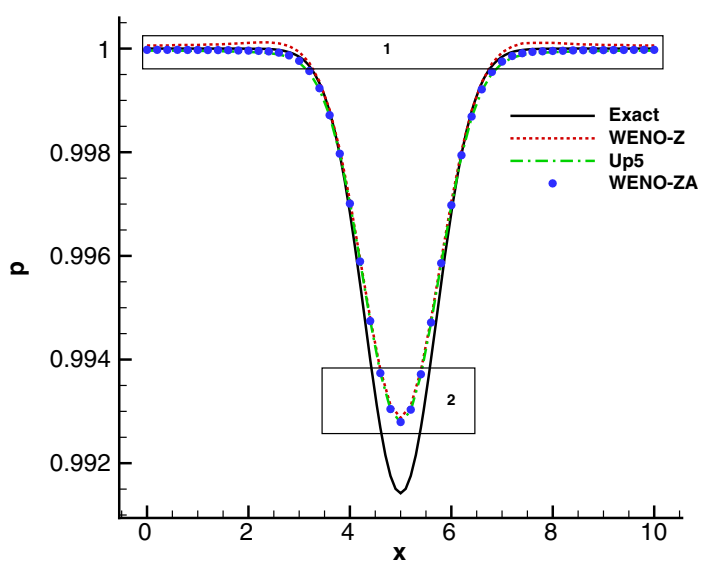

(A)

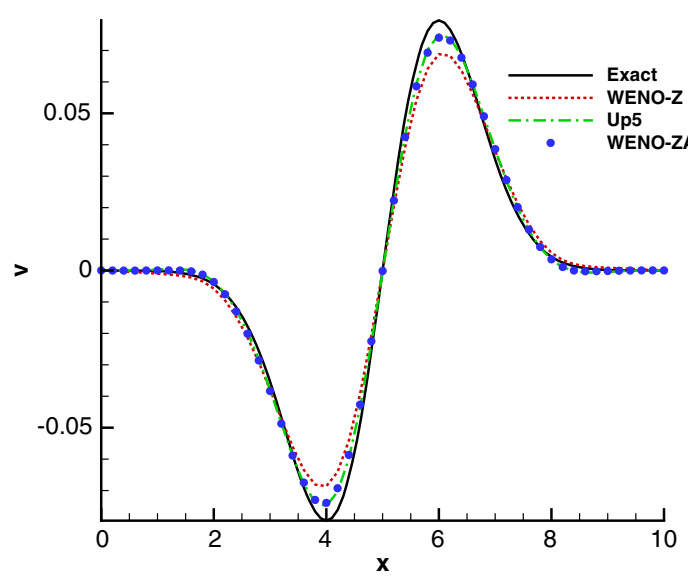

(C)

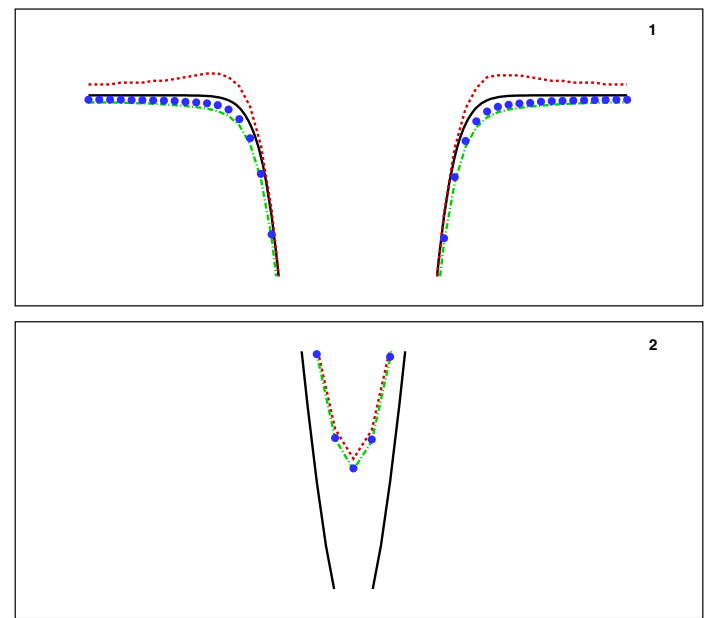

(B)

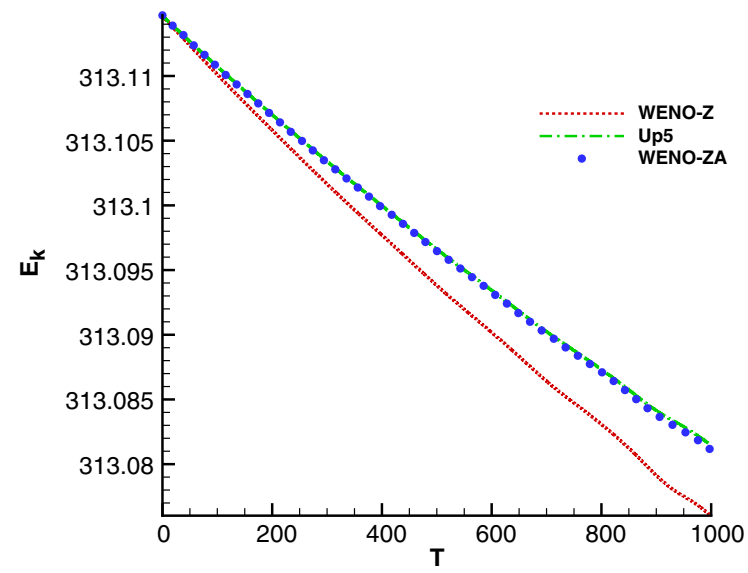

(D)

FIGURE 17 The distribution of pressure (A, B) and velocity $v(\mathrm{C})$ along $y=5$ and the evolution of kinetic energy $E_{k}$ (D). Up5, fifth-order upstream; WENO, weighted essentially nonoscillatory [Colour figure can be viewed at wileyonlinelibrary.com] 
where $(\bar{x}, \bar{y})=(x-5, y-5), r^{2}=\bar{x}^{2}+\bar{y}^{2}$, and the vortex strength $\epsilon=0.5$. The computational domain is $[0,10] \times[0,10]$, and a periodic boundary condition is used in both directions. The results at $t=1000$ with a grid of $50 \times 50$ are shown in Figure 16. Figure 17 gives the distributions of pressure and velocity $v$ along the $x$-axis at $y=5$ and the evolution of kinetic energy $E_{k}$, respectively. The kinetic energy is calculated by $E_{k}=\sum_{i, j=(1,1)}^{N, M} \rho_{i, j}\left(u_{i, j}^{2}+v_{i, j}^{2}\right) / 2$. From these figures, it can be seen that the WENO-ZA scheme performs as well as the upwind scheme (Up5) whereas the WENO-Z scheme shows larger dissipation.

\subsection{2 | Case 8: Riemann problem}

This case is a 2-dimensional Riemann problem ${ }^{9,21}$ with the initial conditions

$$
(\rho, u, v, p)= \begin{cases}(1.5,0,0,1.5), & 0.8 \leq x \leq 1,0.8 \leq y \leq 1, \\ (0.5323,1.206,0,0.3), & 0 \leq x<0.8,0.8 \leq y \leq 1, \\ (0.138,1.206,1.206,0.029), & 0 \leq x<0.8,0 \leq y<0.8, \\ (0.5323,0,1.206,0.3), & 0.8 \leq x \leq 1,0 \leq y<0.8 .\end{cases}
$$

Two set meshes of $200 \times 200$ and $400 \times 400$ are used. The density contours at $t=0.8$ are shown in Figure 18 . It can be seenF18 that both WENO-Z and WENO-ZA schemes can capture reflection shocks and contact discontinuities well. However, the WENO-ZA scheme can resolve the roll-up of the Kelvin-Helmholtz instability with finer structures than the WENO-Z scheme.
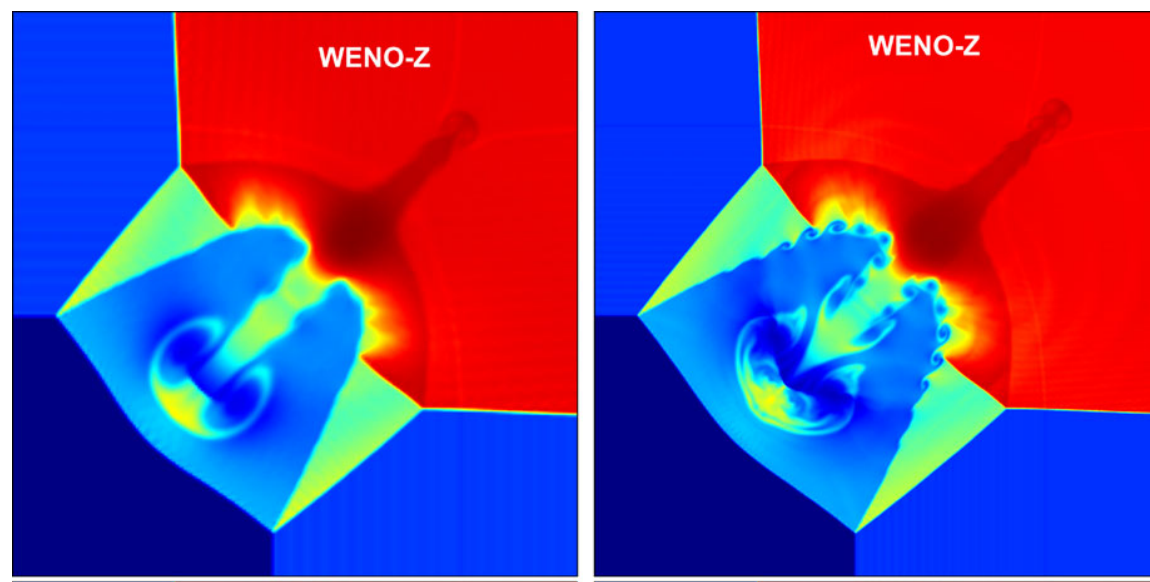

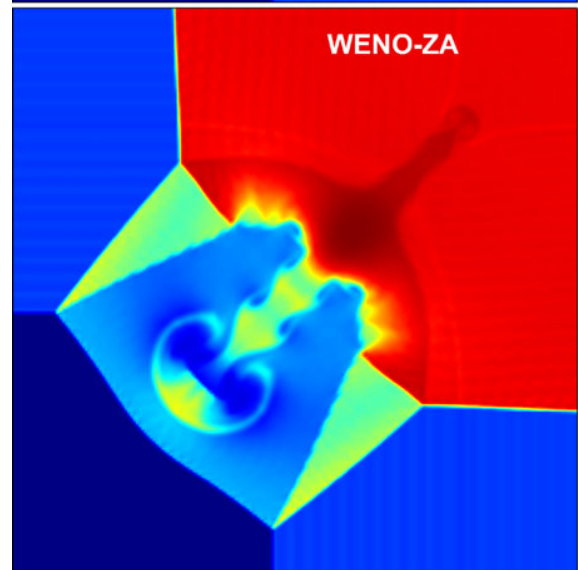

(A) The solutions with $200 \times 200$

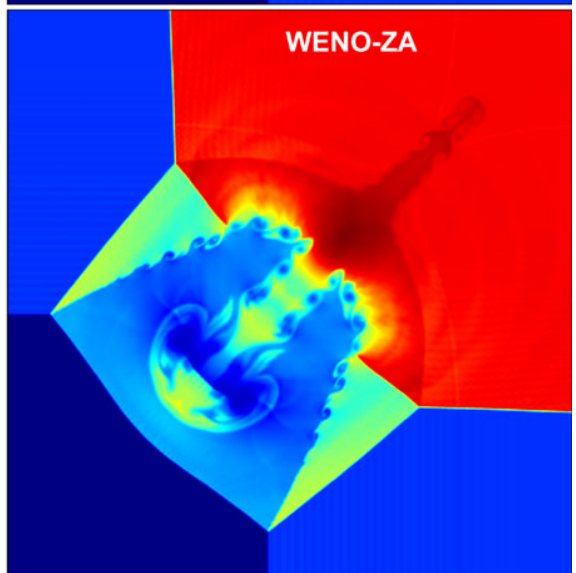

(B) The solutions with $400 \times 400$

FIGURE 18 Density contours of the Riemann problem. WENO, weighted essentially nonoscillatory [Colour figure can be viewed at wileyonlinelibrary.com] 


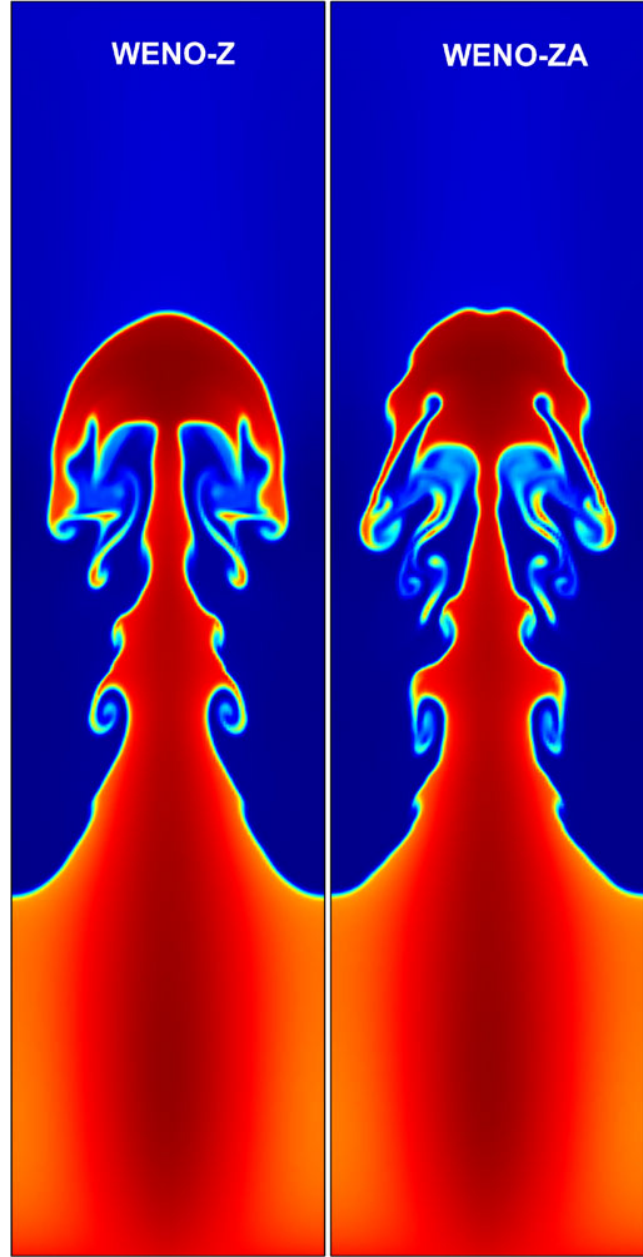

(A) The solutions with $100 \times 400$

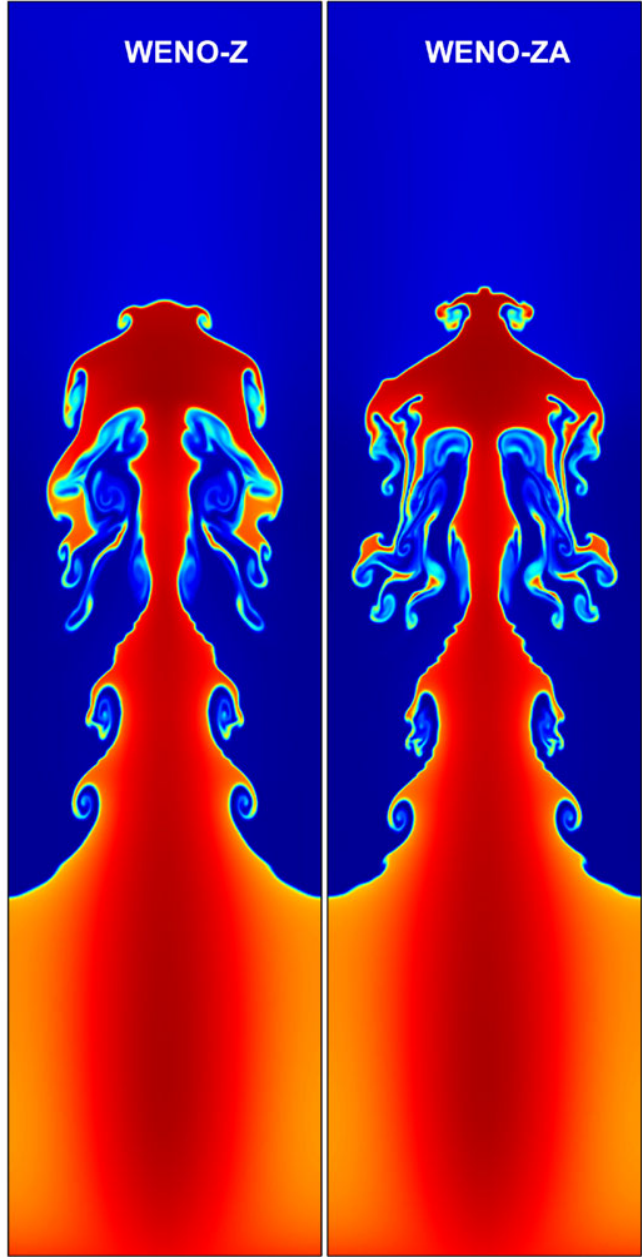

(B) The solutions with $200 \times 800$

FIGURE 19 Density contours of the Rayleigh-Taylor instability problem. WENO, weighted essentially nonoscillatory [Colour figure can be viewed at wileyonlinelibrary.com]

\subsection{3 | Case 9: Rayleigh-Taylor instability problem}

The 2-dimensional Rayleigh-Taylor instability problem ${ }^{22,23}$ is often used to test the numerical dissipation of a high-order scheme. It describes the interface instability between fluids with different densities when acceleration is directed from the heavy fluid to the light one. The gravitational effect is introduced by adding $\rho$ and $\rho v$ to the flux of the $y$-momentum and the energy equations, respectively. The initial distribution is

$$
(\rho, u, v, p)= \begin{cases}(2,0,-0.025 \alpha \cos (8 \pi x), 2 y+1), & 0 \leq y<1 / 2, \\ (1,0,-0.025 \alpha \cos (8 \pi x), y+3 / 2), & 1 / 2 \leq y \leq 1,\end{cases}
$$

and $\alpha=\sqrt{\gamma p / \rho}$ is the speed of sound with $\gamma=5 / 3$. The computational domain is $[0,0.25] \times[0,1]$. The left and right boundaries are reflective boundary conditions, and the top and bottom boundaries are set as $(\rho, u, v, p)=(1,0,0,2.5)$ and $(\rho, u, v, p)=(2,0,0,1)$, respectively. The solution at $t=1.95$ is solved with 2 meshes of $100 \times 400$ and $200 \times 800$. The density contours are plotted in Figure 19. Similar as observed in the previous case, due to its less dissipation, the present scheme generates more complex instability structures than the WENO-Z scheme.

\section{5 | CONCLUSION REMARKS}

To improve accuracy at critical points and keep low dissipation for the WENO-Z scheme, this paper has proposed a sixth-order GSI (GSI-6) and a function $A$ about GSI-6 and the classical LSIs $\left(I S_{k}\right)$. Using the multiplication of GSI-6 and 
the function to calculate the weights, the sufficient condition for fifth-order convergence in smooth regions including critical points is satisfied. Meanwhile, for a discontinuous global stencil, the weights assigned to those substencils containing discontinuities are almost as large as those of the original WENO-Z scheme with power $q=1$; hence, low dissipation is also maintained.

In fact, the multiplication of GSI- 6 and the proposed function $A$ can be regarded as an eighth-order GSI (GSI-8). GSI-8 has the same dimension as the LSIs $\left(I S_{k}\right)$, and there is no any problem-dependent parameter or grid spacing introduced. Hence, the proposed scheme with GSI-8 is more robust than most improved fifth-order WENO-Z schemes.

Furthermore, it is easy to extend the new idea to the third-order or higher-order WENO-Z-type schemes to improve their resolution.

\section{ACKNOWLEDGEMENTS}

This research work was supported by the National Key R\&D Program of China (grant 2016YFA0401200), the National Natural Science Associate Foundation (grant U1530145), the Science Challenge Project (grant TZ2016002), and the National Natural Science Foundation of China (grant 11272325).

\section{ORCID}

\section{Shengping Liu (D) http://orcid.org/0000-0001-9026-0436}

\section{REFERENCES}

1. Shu C-W. High order WENO and DG methods for time-dependent convection-dominated PDEs: a brief survey of several recent developments. J Comput Phys. 2016;316:598-613.

2. Liu X-D, Osher S, Chan T. Weighted essentially non-oscillatory schemes. J Comput Phys. 1994;115(1):200-212.

3. Jiang G-S, Shu C-W. Efficient implementation of weighted ENO schemes. J Comput Phys. 1996;126(1):202-228.

4. Harten A. High resolution schemes for hyperbolic conservation laws. J Comput Phys. 1983;49(3):357-393.

5. Balsara DS, Shu C-W. Monotonicity preserving weighted essentially non-oscillatory schemes with increasingly high order of accuracy. J Comput Phys. 2000;160(2):405-452.

6. Henrick AK, Aslamm TD, Powers JM. Mapped weighted essentially non-oscillatory schemes: achieving optimal order near critical points. J Comput Phys. 2005;207(2):542-567.

7. Borges R, Carmona M, Costa B, Don WS. An improved weighted essentially non-oscillatory scheme for hyperbolic conservation laws. J Comput Phys. 2008;227(6):3191-3211.

8. Castro M, Costa B, Don WS. High order weighted essentially non-oscillatory WENO-Z schemes for hyperbolic conservation laws. J Comput Phys. 2011;230(5):1766-1792.

9. Ha Y, Kim CH, Lee YJ, Yoon J. An improved weighted essentially non-oscillatory scheme with a new smoothness indicator. J Comput Phys. 2013;232(1):68-86.

10. Kim $\mathrm{CH}$, Ha Y, Yoon J. Modified non-linear weights for fifth-order weighted essentially non-oscillatory schemes. $J$ Sci Comput. 2016;67(1):299-323.

11. Fan P, Shen Y, Tian B, Yang C. A new smoothness indicator for improving the weighted essentially non-oscillatory scheme. $J$ Comput Phys. 2014;269:329-354.

12. Hu XY, Wang Q, Adams NA. An adaptive central-upwind weighted essentially non-oscillatory scheme. J Comput Phys. 2010;229(23):8952-8965.

13. Acker F, Borges RBR, Costa B. An improved WENO-Z scheme. J Comput Phys. 2016;313:726-753.

14. Shen YQ, Zha GC. A robust seventh-order WENO scheme and its application. Paper presented at: 46th AIAA Aerospace Sciences Meeting and Exhibit; 2008; Reno, Nevada.

15. Pirozzoli S. On the spectral properties of shock-capturing schemes. J Comput Phys. 2006;219(2):489-497.

16. Shu C-W, Osher S. Efficient implementation of essentially non-oscillatory shock-capturing schemes. J Comput Phys. 1988;77(2):439-471.

17. Shu C-W. Essentially non-oscillatory and weighted essentially non-oscillatory schemes for hyperbolic conservation laws. Advanced Numerical Approximation of Nonlinear Hyperbolic Equations. Berlin Heidelberg: Springer-Verlag; 1997.

18. Roe PL. Approximate Riemann solvers, parameter vectors, and difference schemes. J Comput Phys. 1981;43(2):357-372.

19. Sun Y, Wang ZJ. Evaluation of discontinuous Galerkin and spectral volume methods for scalar and system conservation laws on unstructured grids. Int J Numer Methods Fluids. 2004;45(8):819-838.

20. Davoudzadeh F, McDonald H, Thompson BE. Accuracy evaluation of unsteady CFD numerical schemes by vortex preservation. Comput Fluids. 1995;24(8):883-895.

21. Balsara DS. Multidimensional HLLE Riemann solver: application to Euler and magnetohydrodynamic flows. $J$ Fluid Mech. 2010;229(6):1970-1993. 
22. Shi J, Zhang Y-T, Shu C-W. Resolution of high order WENO schemes for complicated flow structures. J Comput Phys. 2003;186(2):690-696.

23. Yong YN, Tufo H, Dubey A, Rosner R. On the miscible Rayleigh-Taylor instability: two and three dimensions. $J$ Fluid Mech. 2001;447:337-408.

How to cite this article: Liu S, Shen Y, Zeng F, Yu M. A new weighting method for improving the WENO-Z scheme. Int J Numer Meth Fluids. 2018;87:271-291. https://doi.org/10.1002/fld.4490 